\title{
Stability of rotating scalar boson stars with nonlinear interactions
}

\author{
Nils Siemonsen ${ }^{1,2, *}$ and William E. East ${ }^{1}$ \\ ${ }^{1}$ Perimeter Institute for Theoretical Physics, Waterloo, Ontario N2L 2Y5, Canada \\ ${ }^{2}$ Department of Physics 83 Astronomy, University of Waterloo, Waterloo, ON N2L 3G1, Canada
}

(Dated: Tuesday $16^{\text {th }}$ February, 2021)

\begin{abstract}
We study the stability of rotating scalar boson stars, comparing those made from a simple massive complex scalar (referred to as mini boson stars), to those with several different types of nonlinear interactions. To that end, we numerically evolve the nonlinear Einstein-Klein-Gordon equations in $3 \mathrm{D}$, beginning with stationary boson star solutions. We show that the linear, non-axisymmetric instability found in mini boson stars with azimuthal number $m=1$ persists across the entire parameter space for these stars, though the timescale diverges in the Newtonian limit. Therefore, any boson star with $m=1$ that is sufficiently far into the non-relativistic regime, where the leading order mass term dominates, will be unstable, independent of the nonlinear scalar self-interactions. However, we do find regions of $m=1$ boson star parameter space where adding nonlinear interactions to the scalar potential quenches the non-axisymmetric instability, both on the non-relativistic, and the relativistic branches of solutions. We also consider select boson stars with $m=2$, finding instability in all cases. For the cases exhibiting instability, we follow the nonlinear development, finding a range of dynamics including fragmentation into multiple unbound non-rotating stars, and formation of binary black holes. Finally, we comment on the relationship between stability and criteria based on the rotating boson star's frequency in relation to that of a spherical boson star or the existence of a co-rotation point. The boson stars that we find not to exhibit instability when evolved for many dynamical times include rapidly rotating cases where the compactness is comparable to that of a black hole or neutron star.
\end{abstract}

\section{INTRODUCTION}

Gravitational wave (GW) astronomy [1-3] and electromagnetic observations [4-6] have shown that the black hole $(\mathrm{BH})$ paradigm can be used to explain phenomena ranging from the merger and ringdown of compact object binaries, to active galactic nuclei. However, while this paradigm explains physics across several orders of magnitude extremely successfully, the BH's defining featurethe event horizon - cannot be directly probed experimentally. Additionally, some quantum gravity models predict strong deviations from general relativity approaching the boundaries of BHs, and extensions to the Standard Model can lead to the formation of highly compact BH-like objects. This has motivated the construction of a large class of exotic compact objects (ECOs), with various physical and mathematical motivations, mimicking many of the gravitational properties attributed to classical $\mathrm{BH}$ spacetimes, and providing an alternative to the $\mathrm{BH}$ paradigm.

Beside BHs and neutron stars, a large class of ECOs has been conceived [7]. The compactness of ordinary fluid stars, like neutron stars, is restricted by the Buchdahl limit [8] for spherically symmetric configurations, and more stringently by the requirement that the sound speed be less than the speed of light. However, these objects could capture and accrete dark matter from their environment, forming a composite object [9-14], which could exceed these compactness limitations. Various models of ordinary matter also predict the existence of anisotropic stars with compactness levels arbitrarily close to those

*nsiemonsen@perimeterinstitute.ca of BHs [15-17]. String theory inspired solutions, called fuzzballs, emerge as averages over microstates, generating horizonless, but highly compact, BH-like models [18-22]. There is speculation that quantum effects of a collapsing horizonless spacetime could halt the complete classical collapse to a $\mathrm{BH}$ and yield a highly compact configuration just outside the BH-limit [23-26]. Furthermore, ultralight scalar or vector particles arise in compactifications of string theory, solutions to the strong CP-problem [27, 28], as well as as ultralight and fuzzy dark matter [29-34]. If such particles exist in the universe, stationary boson stars (BSs) [35-42] can be formed through a gravitational cooling mechanisms out of a diffuse distribution of bosonic matter [37, 43]. Lastly, there is an extended class of other ECOs like gravastars, wormholes, or firewalls [7]. The common feature of all these approaches is a certain "closeness," measured by the compactness and related features, to classical BHs.

Accurate predictions of the properties of $\mathrm{BH}$ mimickers, especially of their dynamics in the nonlinear regime in the case of mergers, is needed to confirm or disfavor the existence of BHs using $\mathrm{GW}$ or electromagnetic observations [44-49]. However, for many ECO models, determining their nonlinear evolution is challenging or ill-posed, due to their vastly different physical and mathematical origins. BSs, on the other hand, obeying standard energy conditions (as long as their potential is non-negative), evolve according to well behaved wave-like equations, and can be treated numerically using the same techniques as the Einstein equations. Thus they provide a simple and tractable setting to explore dynamical properties of ECOs and BH mimickers $[41,50]$. Stationary rotating BSs can have compactnesses approaching that of BHs, 
and therefore, capture the main gravitational features of a large set of ECOs. BSs can exhibit an innermost stable circular orbit, and unstable and stable photon orbits while being horizonless and regular everywhere. Therefore, these solutions provide an ideal test bed to study the nonlinear dynamics of a class of ultracompact objects.

However, while there is an extensive literature constructing stationary BS solutions in general relativity, the number of studies that have looked at the dynamics or stability of these objects is more limited, in particular for BSs with angular momentum (see Ref. [41] for a recent review.) Recently, there has been increasing interest in studying the nonlinear dynamics of scalar and vector BSs in various scenarios (e.g. Refs. [51-58] and see Ref. [41]). And in the past few years, several studies have presented evidence that rotating scalar BSs might be inherently unstable. In Refs. [54, 59], the inspiral and merger of binary scalar BSs settled, above a certain critical BH-threshold, into a non-rotating scalar BSs in the final state, shedding all angular momentum in the process. Similar results were found for the collapse of rotating clouds of scalar field [60]. Furthermore, Ref. [43] considered a number of rotating scalar BSs made of massive bosons without interactions, and found them all to be subject to a non-axisymmetric instability (NAI), rendering them little use, e.g., for studying the dynamics of a merger.

However, in this work, we show that this problem can be cured by considering nonlinear interactions for the scalar field, and present evidence for the stability of a large class of rotating BSs both in the relativistic (high compactness) and in the non-relativistic (dilute) regime. In particular, we show that when considering parameterized families of BSs for several different choices of the scalar field potential, the growth rate for the NAI approaches zero at certain critical values (e.g., of the frequency of the BS). Nonlinearly evolving select cases beyond these critical values for many dynamical times, we find no evidence for instability. (Though our methods do not allow us to rule out some instability operating on even longer timescales.) Hence, such rotating scalar BS solutions are promising candidates for studying the dynamics of isolated and binary ECOs in a nonlinear scenario, and comparing to BHs.

In Sec. II, we outline the scalar field models we consider, describe the numerical techniques we use to construct rotating BS solutions in these models, and review the linear stability results in the literature. Following this, we present our numerical results in Sec. III. We first identify the form and nature of the NAI in the linear regime, then measure the growth rates of the NAI for a set of BSs and potentials, and present select isolated rotating BSs that show no sign of instability. We also analyze the final state of the instability, and discuss various physical explanations for the onset of instability. Finally, we conclude in Sec. IV. Additional details on the numerical methods and error estimates from convergence studies are given in the appendices. In the following, we

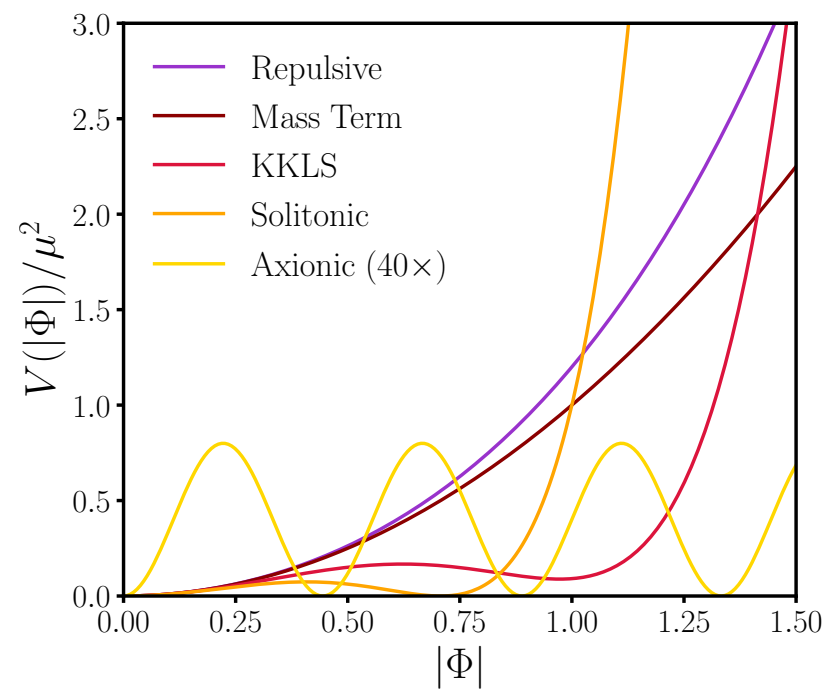

Figure 1. Examples of the nonlinear self-interactions, specified by $V(|\Phi|)$, considered here. We emphasize the characteristic features of each: The solitonic potential has a non-trivial vacuum state, the KKLS self-interaction has a metastable state, the axionic potential is periodic, and finally, the repulsive potential has a positive first correction to the mass term.

use units with $G=c=1$ and the $(-+++)$ metric signature.

\section{STATIONARY SCALAR BOSON STARS}

\section{A. Models considered}

In this work, we consider rotating scalar BSs in minimally coupled massive complex scalar models, comparing stars where the scalar potential only has a mass term (referred to as mini BSs) to stars where the potential has additional higher order terms, corresponding to nontrivial scalar self-interactions. The latter are motivated by axion-like particles, effective field theory descriptions of light scalar degrees of freedom, or simply by the finding that such models can produce highly compact BSs. As such, we consider a complex scalar field $\Phi$, minimally coupled to the Einstein-Hilbert action, exhibiting a global $\mathrm{U}(1)$ symmetry:

$$
S=\int d^{4} x \sqrt{-g}\left[\frac{R}{16 \pi}-g^{\alpha \beta} \nabla_{(\alpha} \bar{\Phi} \nabla_{\beta)} \Phi-V(|\Phi|)\right] .
$$

Here $R$ is the curvature scalar, the overbar denotes complex conjugation, and the potential $V(|\Phi|)$ contains both the mass term and nonlinear self-interactions of the complex scalar field. All such self-interactions considered here (see also Figure 1) reduce to the mass term ${ }^{1}$,

\footnotetext{
1 The scalar mass $m_{\Phi}$ and the mass parameter $\mu$ are related by $\mu=m_{\Phi} / \hbar$
} 
$V(|\Phi|) \rightarrow \mu^{2}|\Phi|^{2}$, in a small-coupling limit.

Solitonic potential: This potential is characterized by a single additional coupling parameter $\sigma$, such that [61]

$$
V(|\Phi|)=\mu^{2}|\Phi|^{2}\left(1-\frac{2|\Phi|^{2}}{\sigma^{2}}\right)^{2},
$$

reduces to the mass-term if $\sigma \rightarrow \infty$. As indicated in Figure 1 , this potential has a negative first correction in the expansion in $|\Phi|$, and features a non-trivial vacuum state at $|\Phi|=\sigma / \sqrt{2}$. With these self-interactions, localized scalar field configurations (i.e., non-topological solitons) exist even in the absence of gravity [61], and spherically symmetric BSs of this type are sufficiently compact to support stable trapped null geodesics [54].

KKLS potential: The potential [62, 63],

$$
V(|\Phi|)=\mu^{2}|\Phi|^{2}\left[1-\frac{16 \pi}{1.1 \kappa}|\Phi|^{2}+\frac{64 \pi^{2}}{1.1 \kappa^{2}}|\Phi|^{4}\right],
$$

is parameterized by $\kappa$ (see also Ref. [64]). It also exhibits a negative first correction beyond the mass-term, and a local minimum at a non-zero value of $|\Phi|$ (see also Figure 1). The KKLS potential simplifies to the mass term if $\kappa \rightarrow \infty$ [62]. After rescaling $|\Phi| \rightarrow(\kappa /(8 \pi))^{1 / 2}|\Phi|$, BSs in this model reduce to non-gravitating non-topological Q-balls [65] in the $\kappa=0$ limit.

Axionic potential: Inspired by ultralight particles predicted, for instance, by string theory compactifications, or to solve the QCD CP-problem, we consider a generic axion-like potential of the form

$$
V(|\Phi|)=\mu^{2} f^{2}\left\{1-\cos \left[\sqrt{2|\Phi|^{2}} f^{-1}\right]\right\} .
$$

This periodic potential is parameterized by the coupling $f$, reduces to the mass term if $f \rightarrow \infty$, and has a negative first correction, when expanded in small $|\Phi|$.

Repulsive potential: While all the above scalar selfinteractions have attractive (i.e., negative) first corrections to the mass term, we also study the effects of a non-trivial repulsive first correction of the form

$$
V(|\Phi|)=\mu^{2}|\Phi|^{2}+\lambda|\Phi|^{4}
$$

with $\lambda>0$. In the following, we also briefly comment on the properties of BSs in a scalar model with a Liouville potential [66, 67],

$$
V(|\Phi|)=\mu^{2} \alpha^{2}\left[e^{|\Phi|^{2} / \alpha^{2}}-1\right]
$$

which has the same form as Eq. (5) when expanded to quadratic order in $|\Phi|^{2}$.

\section{B. Stationary solutions and classical observables}

We consider BS solutions where the spacetime is regular, stationary, axisymmetric, and asymptotically flat.
In Lewis-Papapetrou coordinates, the metric takes the general form

$$
\begin{aligned}
d s^{2}=-f d t^{2}+l f^{-1} & \left\{g\left(d r^{2}+r^{2} d \theta^{2}\right)\right. \\
+ & \left.r^{2} \sin ^{2} \theta\left(d \varphi-\Omega r^{-1} d t\right)^{2}\right\} .
\end{aligned}
$$

Here all metric functions $\{f, l, g, \Omega\}$ depend only on $(r, \theta)$, in accordance with our assumptions. The complex scalar field is assumed to have the form $\Phi=e^{i \omega t+i m \varphi} \phi(r, \theta)$, with $\phi \in \mathbb{R}$. This time and azimuthal dependency ensures that the scalar field stress-energy tensor is stationary and axisymmetric (maintaining the symmetry of the metric). In addition to the above five physical fields, we introduce two auxiliary fields $\rho(r, \theta)$ and $\omega_{s}(r, \theta)$, following Ref. [62], which aid in imposing certain conditions on the BSs (see appendix A for details). With this ansatz, in conjunction with regularity conditions at the origin and asymptotic flatness conditions at infinity, the coupled system of Einstein-complex-Klein-Gordon equations

$$
G_{\mu \nu}=8 \pi T_{\mu \nu}, \quad \nabla_{\alpha} \nabla^{\alpha} \Phi+\Phi \partial_{|\Phi|^{2}} V(|\Phi|)=0
$$

[together with Eq. (A3)] reduce to an elliptic boundary value problem. Given a sufficiently accurate initial seed for a Newton-Raphson type relaxation scheme, the parameter space is explored by marching along the respective parameters. We give more details on how we numerically solve these equations to construct BS solutions in Appendix A.

In asymptotically flat, stationary spacetimes, the Komar mass integrated over a sphere at spatial infinity, and the ADM mass of a spatial slice $\Sigma_{t}$ coincide. Both can be written as

$$
M=\int_{D} d r d \theta d \varphi \sqrt{-g}\left(2 T_{t}^{t}-T_{\alpha}^{\alpha}\right)
$$

where the integral is over the Lewis-Papapetrou spatial coordinate domain $D$. The axisymmetry and regularity of Eq. (7) ensures that the total angular momentum of the spacetime is due to the scalar field through

$$
J=-\int_{D} d r d \theta d \varphi \sqrt{-g} T_{\varphi}^{t} .
$$

Finally, the global U(1) symmetry of the complex scalar theory gives rise to a conserved $\mathrm{U}(1)$-Noether charge $Q$ that measures the particle number (i.e., the occupation number of the Bose-Einstein condensate ${ }^{2}$ ). The associated Noether current is

$$
j^{\mu}=-i\left(\bar{\Phi} \partial^{\mu} \Phi-\Phi \partial^{\mu} \bar{\Phi}\right), \quad \nabla_{\mu} j^{\mu}=0 .
$$

Following immediately from noticing that $T_{\varphi}^{t}=m j^{t}$ (in terms of the Noether current Eq. (11)), the angular

\footnotetext{
2 When quantizing the complex scalar field theory, the charge $Q$ counts the (anti)-particles of a given state, making the relation manifest.
} 

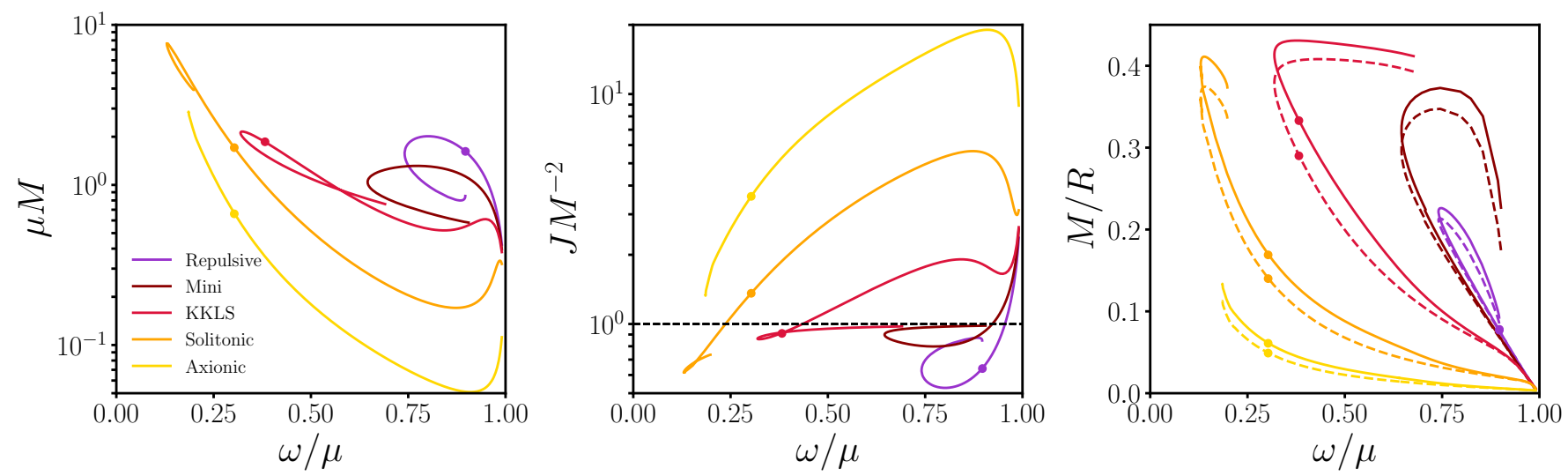

Figure 2. We present the main properties of five families of $m=1$ rotating BSs in the above scalar models, for choices of coupling focused on in this work. The solitonic BS family has coupling $\sigma=0.05$, the axionic family has coupling $f=0.005$, the BSs in the KKLS model have $\kappa=0.1$, the family of BSs with repulsive self-interactions have coupling $\lambda / \mu^{2}=5 \times 10^{2}$, while the mini BS family is given for reference. From left to right, the three plots show the total mass $M$ in units of $\mu^{-1}$, the dimensionless spin $J / M^{2}$, and the compactness $M / R$ of each of these families of BSs as a function of the boson's frequency $\omega / \mu$. For determining the radius of the BSs in the last plot, we use either the surface containing $99 \%$ of the mass $C^{K}$ (solid), or $99 \%$ of the U(1)-charge density $C^{Q}$ (dashed), as discussed in Sec. II B. Based on the turning point argument described in the text, BSs past the maximum of the total mass are expected to be unstable. Notice that in the non-relativistic limit, i.e., when $\omega / \mu \rightarrow 1$, the behavior of all families of BSs with non-trivial self-interactions reduce to that of mini BSs [68, 69]. The individual data points indicate BSs that we evolve and where we find no sign of an instability (see Sec. III B). Note that, although not evident in the plot, the axionic family reaches a global maximum of $\mu M$ at $\omega / \mu \approx 0.187$.

momentum of the system is "quantized" into integer increments of the scalar charge, $J=m Q$, dictated by the azimuthal number $m$. Of course, this relation is a purely classical constraint, but hints towards the quantum interpretation of the system as a coherent condensate of a set of bosons.

At sufficiently large radial coordinate, the scalar BS solution exhibits an exponential tail, $\phi \sim \exp (-\beta r)$, for some $\beta>0$, making any notion of a radius for the star ambiguous. In the non-rotating case, the radius of a BS is typically defined by the areal radius, $R_{99}$, at which $99 \%$ of the BS's Komar mass Eq. (9) is contained within a coordinate 2 -sphere of radius $R_{99}$. For rotating BSs, we introduce two distinct notions of size: (i) We define the $R_{99}^{K}$ as the circular radius $^{3} \tilde{r}$, for which $M(\tilde{r})=0.99 M(\tilde{r} \rightarrow \infty)$, and (ii) we define $R_{99}^{Q}$ as the circular radius, for which $Q(\tilde{r})=0.99 Q(\tilde{r} \rightarrow \infty)$. Note that, while $M$ contains both the scalar and the gravitational binding energy, $Q$ measures only the scalar rest mass. Based on this notion of size, the compactness of a rotating BS is given either by $C^{K}=M / R_{99}^{K}$ or $C^{Q}=M / R_{99}^{Q}$. For comparison, for a non-rotating $\mathrm{BH} C_{\mathrm{BH}}^{K}=1 / 2$, while for a typical neutron $\operatorname{star} C_{\mathrm{NS}}^{K} \sim 0.1$. For any given BS, the difference between $C^{Q}$ and $C^{K}$ is indicative of the ambiguity in defining its radius.

In Figure 2, we present these observables for several sets of families of solutions. The Newtonian limit is approached as the gravitational binding energy, of order

3 The radius $\tilde{r}$ for which the proper length of a circle $C$ in the equatorial plane is $C=2 \pi \tilde{r}$.
$M / R$, is small. In that limit, the bosons' frequency $\omega$ approaches $\mu$, the marginally bound value, since $\omega / \mu-1 \sim$ $M / R$.

\section{Stability arguments}

The stability of non-rotating BSs has been investigated by means of (i) analyzing the temporal dependence of individual modes or more general linear perturbations [70-72], (ii) applying catastrophe theory or thermodynamic stability to families of BS solutions [64, 73-76], and (iii) evolving the Einstein-Klein-Gordon system of equations numerically to study the nonlinear stability of BSs [41, 77-80]. The consensus of these methods is that spherically symmetric BSs switch their radial stability properties whenever the BS's mass reaches an extremum as a function of the central scalar field value $\phi(0)$ : $d M / d \phi(0)=0$. As such, non-rotating BSs have at least one stable branch reaching from the non-relativistic (i.e., dilute) limit, to the first maximum of $M$. Depending on the potential, non-rotating BSs can have a number of stable branches, analogous to the white dwarf and neutron star branches of fluid stars.

However, the stability analysis of rotating BSs is considerably more complex ${ }^{4}$. Even at the linear level, there is not expected to be a clean decoupling of the scalar

\footnotetext{
${ }^{4}$ Here we will not consider BSs with ergoregions, which would be subject to the ergoregion instability [81].
} 
and the gravitational modes, making a linear stability analysis difficult. Based on turning point arguments in Ref. [64] (see also Ref. [82] for a brief analysis of the stability of excited BSs with the KKLS potential), the stability of rotating BSs should switch, analogous to non-rotating BSs, at the extrema of $M(\omega)$. In Figure 2 , we present the total mass, angular momentum, and compactness of five different potentials and families of $m=1$ fundamental BSs. Applying the arguments above to these families of BSs, we see that mini BSs and those in the repulsive scalar model Eq. (5) exhibit a single (potentially) stable branch reaching from the non-relativistic limit, $\omega / \mu \lesssim 1$, to the frequency where $M$ (or equivalently $Q$ ) reaches the global maximum, while all solutions past that maximum are unstable. Similarly, the family of BSs in the axionic, solitonic, and KKLS scalar models exhibit two distinct potentially stable branches. Since all these potentials reduce to the mass term in the non-relativistic limit, it is not surprising that BSs with these self-interactions are stable in the non-relativistic limit based on the turning point arguments. However, these BSs have another potentially stable branch in the relativistic (high-compactness) regime, between the first local minimum of $M$ and its global maximum, where $\partial M / \partial \omega<0$.

However, as seen, for instance, in Refs. [75, 76], turning points are only a sufficient condition for the existence of a thermodynamic instability, not a necessary condition. In fact, a thermodynamic instability could appear without the presence of a turning point. On top of this, thermodynamically unstable systems are not necessarily dynamically unstable: While there exists a preferred solution, there may not be a path through the solutions space that is dynamically achievable. Given these arguments, and the fact that some BSs are dynamically unstable in a regime not indicated by turning point arguments [43], a more detailed analysis of the dynamical nonlinear stability is necessary.

\section{RESULTS}

In contrast to the stability analysis outlined in the last section, in Ref. [43], it was discovered that $m=$ 1 scalar mini BSs are subject to a NAI in a regime where they are expected to be stable based on turning point arguments. In those cases, the evolution of the perturbed stationary scalar BSs revealed exponentially growing non-axisymmetric modes whose nonlinear evolution ultimately lead to the gravitational collapse of the BS to a BH. In the following, expanding on the work presented in Ref. [43] (see also Ref. [60] for the same instability present in dilute bosonic clouds), we study the nonlinear dynamical behavior of a large class of $m=1$ (and some $m=2$ ) mini BSs and BSs in the models presented in II A. To that end, we numerically evolve the Einstein-Klein-Gordon equations, Eq. (8), in 3D from stationary BS initial data that is perturbed only by nu- merical truncation error (see Appendix B for details of the numerical evolution). In many cases, we find a similar NAI and determine its growth rate as a function of the BS parameters. However, we identify two distinct regions of the parameter space, where the NAI growth rate approaches zero. Past this point, rotating scalar BSs appear to be stable under nonlinear evolution. This is true for parts of the relativistic branch, identified in Sec. II C, as well as portions of the non-relativistic branch. Furthermore, we show that the instability growth rate for BSs in any scalar model, approaches zero (both in units of the BS mass and BS radius) in the dilute/Newtonian limit, where the nonlinear interactions can be neglected, and all BSs reduce to mini BSs.

In Sec. III A, we illustrate the linear characteristics of the NAI and explain how we extract the identifying features. In Sec. III B, we present the growth rates and other results on the unstable linear modes for a number of types of rotating BSs across the parameter space of the BSs' frequency and couplings, as well as explicitly demonstrate the stable evolution of a few example cases. In Sec. III C, we describe the ultimate fate of the unstable BSs, and finally, in Sec. III D, we examine several criteria for characterizing the onset of the NAI in BSs.

\section{A. Characterization of the linear non-axisymmetric instability}

In the linear regime, the NAI manifests as an exponentially growing, non-axisymmetric perturbation to both the scalar field and metric. We study this by evolving stationary BSs solutions in time, letting numerical truncation error seed the instability at a small amplitude that grows by several orders of magnitude before becoming nonlinear. This allows us to characterize the NAI during this extended linear phase.

We will be interested in monitoring perturbations to quantities that are stationary in the background BS solution, which has an axisymmetric and stationary spacetime. For example, linear perturbations to the magnitude squared of the BS complex scalar field $\Phi_{\mathrm{BS}}$ can be written as

$$
|\Phi|^{2}-\left|\Phi_{\mathrm{BS}}\right|^{2}=e^{-i \tilde{\omega} t} e^{i \tilde{m} \varphi} \delta \Phi(r, \theta),
$$

where $\tilde{\omega}=\tilde{\omega}_{R}+i \tilde{\omega}_{I}$ captures both the harmonic time dependence, $\tilde{\omega}_{R}>0$, and possible (un)stable dynamical behavior with $\tilde{\omega}_{I}<0(>0)$, while $\tilde{m}$ is the azimuthal mode number. In the time domain setting, we are assuming ${ }^{5}$ that a single perturbative mode (the most unstable) dominates the dynamics during the linear instability phase. We characterize the NAI by its azimuthal

\footnotetext{
${ }^{5}$ We confirm explicitly that the $\varphi$-dependence of the linear perturbation has large support over only a single azimuthal mode $\tilde{m}$.
} 


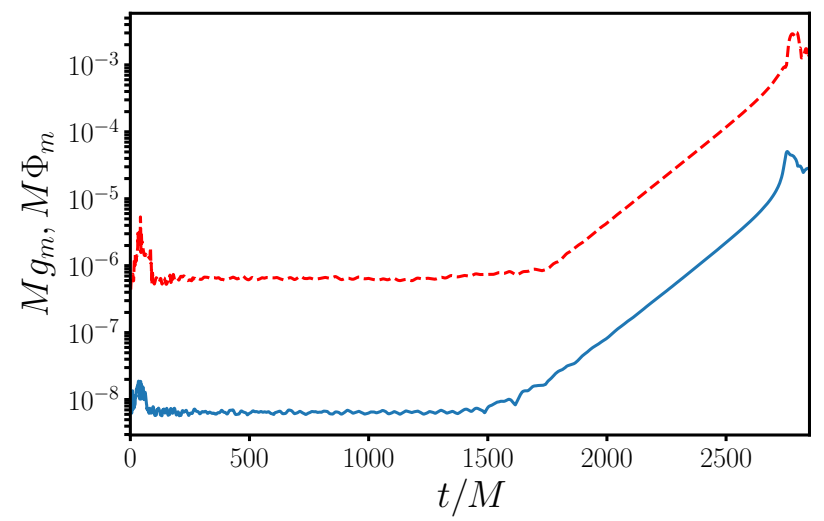

Figure 3. The evolution of the global maxima $g_{m}=$ $\max \left|\partial_{t} g_{t t}\right|$ (red dashed) and $\Phi_{m}=\left.\max \left|\partial_{t}\right| \Phi\right|^{2} \mid$ (blue) [see Eq. (13)] for an axionic $m=1$ BS with $f=5 \times 10^{-2}$ and $\omega / \mu=0.425$. The NAI is evident as a linear instability beginning around $t / M \approx 1.75 \times 10^{3}$, and enters the nonlinear regime at roughly $t / M \approx 2.75 \times 10^{3}$. The perturbations present at early times originate from lower order interpolations at the mesh refinement boundaries (see Appendix B for details).

mode number, complex frequency, as well as the radial and polar dependency encoded in $\delta \Phi(r, \theta)$. We can extract the growth rates $\tilde{\omega}_{I}$ by fitting an exponential to the global maxima

$$
\Phi_{m}:=\left.\max _{x \in \Sigma_{t}}\left|\partial_{t}\right| \Phi\right|^{2} \mid \quad \text { and } \quad g_{m}:=\max _{x \in \Sigma_{t}}\left|\partial_{t} g_{t t}\right|
$$

of a given time slice $\Sigma_{t}$, which quantify the divergence from a stationary solution. In Figure 3, we depict the typical dynamical behavior of $\Phi_{m}$ and $g_{m}$ for an $m=1$ scalar BS in the linear regime.

Furthermore, in Figure 4, we present an example of the difference in magnitude of the scalar field, $\Delta|\Phi|^{2}$, during the linear phase (at $t=t_{\mathrm{NAI}}$ ) of the NAI, compared to the initial data (at $t=0$ ), in the equatorial plane of the BS:

$$
\Delta|\Phi|^{2}:=|\Phi(t=0)|^{2}-\left|\Phi\left(t=t_{\mathrm{NAI}}\right)\right|^{2}
$$

From this, we can extract the azimuthal mode number $\tilde{m}$, the radial dependence, and the harmonic part of the frequency $\tilde{\omega}_{R}$. The last-named is extracted by finding the radius $R_{m}$ where the perturbation is largest, $\max _{x \in \Sigma_{t}} \Delta|\Phi|^{2}=\Delta\left|\Phi\left(r=R_{m}, \theta=\pi / 2\right)\right|^{2}$; we then fit $\cos (\tilde{m} \varphi+\phi(t))$ to $\Delta\left|\Phi\left(R_{m}, \theta=\pi / 2, \varphi\right)\right|^{2}$ as a function of time during the exponential growth phase of the mode. The essentially constant time derivative of the phase $\dot{\phi}$ gives $\tilde{\omega}_{R}$ for the unstable mode. As can be seen in Figure 4 , the azimuthal mode number $\tilde{m}$ is evident from $\Delta|\Phi|^{2}$, and shows large support only over a single mode $\tilde{m}$. These modes could equivalently be extracted from perturbations to the energy density, or other projections of the stress-energy tensor, due to the global U(1) symmetry. In the following section, we present the characteristics of the NAI extracted in this way from our timedomain evolutions for several different families of rotating BSs.

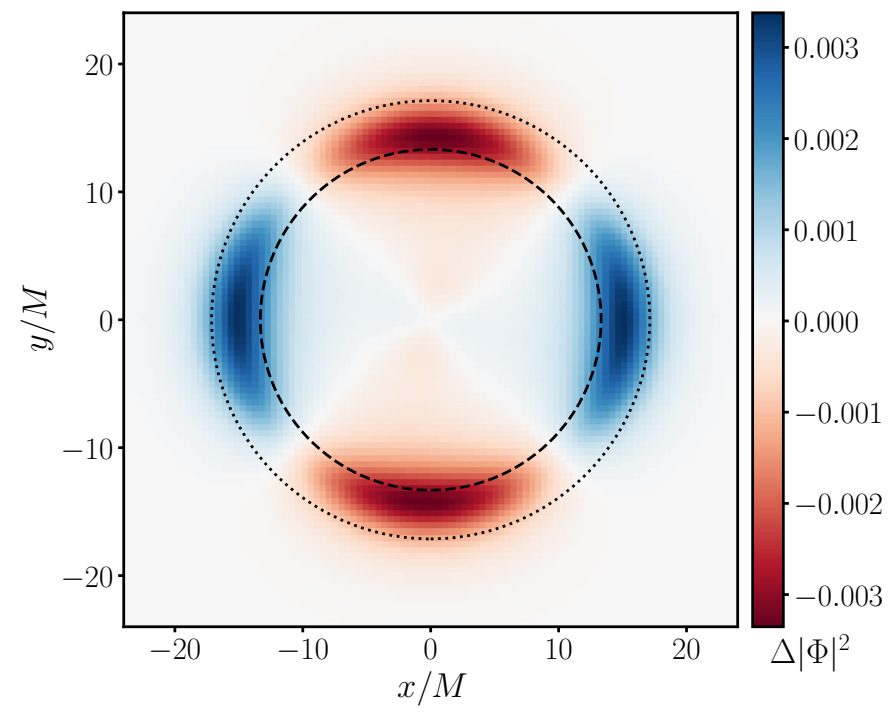

Figure 4. The radial dependence of the unstable mode from a typical case, in particular the axionic BS considered in Figure 3. We show $\Delta|\Phi|^{2}$ [defined in Eq. (14)] in the equatorial plane (parameterized by the Cartesian coordinates $x$ and $y$ ), where we recall that $|\Phi(t=0)|^{2}$ is axisymmetric. This quantity is extracted at $t_{\mathrm{NAI}}=2.5 \times 10^{3} \mathrm{M}$ which, as can be seen from Figure 3, is solidly in the linear instability regime. The depicted structure rotates about the center of the equatorial plane at an angular frequency $\tilde{\omega}_{R} M=2.44 \times 10^{-2}$, while its magnitude grows exponentially with $\tilde{\omega}_{I} M=6.4 \times 10^{-3}$. For reference, we also add the radii $R_{99}^{K}$ (dotted circle) and $R_{99}^{Q}$ (dashed circle) defined in Sec. II B.

\section{B. Results: Instability growth rates}

In the following, we focus on finding the complex frequency $\tilde{\omega}$ for the unstable modes of the NAI. For all the families of BSs presented in Figure 2, we investigate how $\tilde{\omega}_{I}$ changes with the BS frequency or coupling. For selfinteractions with an attractive first correction, we focus on the second potentially stable branch (based on turning point arguments, as discussed in Sec. II C), while for mini BSs and those in models with repulsive potentials, we focus on the only branch that is connected to the non-relativistic limit. In this limit, which corresponds to $\omega \rightarrow \mu$, the scalar field amplitude becomes small, and only the lowest-order term in the potential, i.e. the mass term, will be important. Ultimately, we identify critical points $\omega^{c} / \mu$ and $\lambda^{c} / \mu^{2}$ where $\tilde{\omega}_{I}$ tends toward zero, i.e. the NAI shuts off.

In Figure 5, we show the real and imaginary frequency of the unstable mode for axionic, solitonic, and KKLS scalar rotating BSs with $m=1$, as a function of the BS's frequency $\omega / \mu<0.875$, for fixed coupling constants. All the cases shown exhibit an $\tilde{m}=2 \mathrm{NAI}$ with a positive $\tilde{\omega}_{I}$ in the frequency ranges with $\omega / \mu$ larger than the critical values:

$$
\omega_{\mathrm{A}}^{c} / \mu=0.392, \quad \omega_{\mathrm{S}}^{c} / \mu=0.493, \quad \omega_{\mathrm{K}}^{c} / \mu=0.490 .
$$

Here the subscripts stand for axionic, solitonic, and 


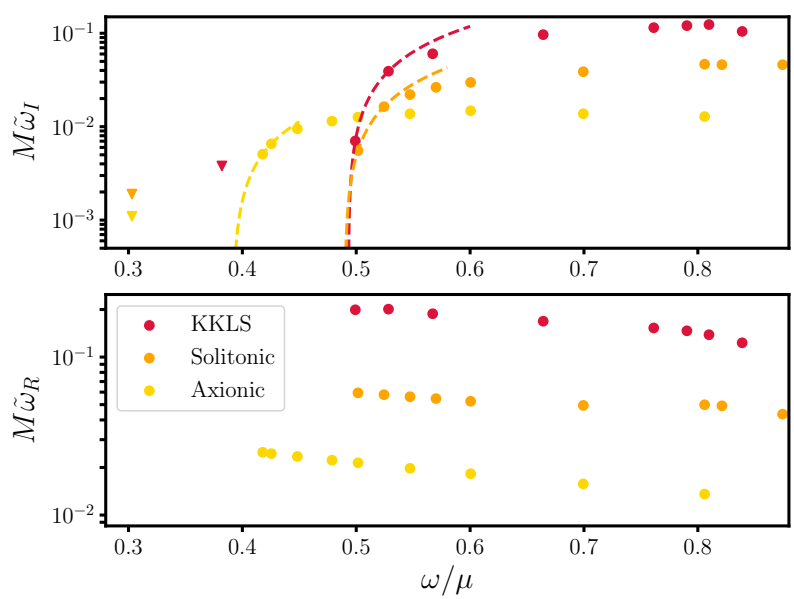

Figure 5. The imaginary (top) and real (bottom) components of the frequency of the $\tilde{m}=2$ unstable mode as a function of the BS frequency $\omega / \mu$ for three families of solutions shown in Figure 2. The axionic model has coupling $f=5 \times 10^{-2}$, the solitonic potential has $\sigma=0.05$, and the KKLS selfinteractions are characterized by $\kappa=0.1$. All three families are sequences of $m=1$ rotating BSs. The dashed curves are linear interpolations considering only the two points with smallest $\omega / \mu$ in each respective case. We also indicate the upper bounds on the growth rate of the cases considered in Table I by down-arrows.

KKLS, respectively. From Figure 2, we see that all the BSs shown in Figure 5 are on the second branch that is nominally stable based on turning point arguments. Approaching these critical values from above, we find that the instability rate $\tilde{\omega}_{I}$ tends toward zero. Though it becomes more and more computationally expensive to measure longer and longer instability timescales, $\tilde{\omega}_{I}$ appears to be approaching zero roughly linearly in $\omega$. This suggests that below this critical value, $\omega<\omega^{c}$ (and above the global maximum of the BS's mass), there is a range of BS solutions in the relativistic regime of the nonlinear interactions models we consider, that are free of the NAI. Again, consulting Figure 2, we see that such solutions have large compactness.

We also study the stability of $m=1$ BSs with the repulsive nonlinear scalar interactions given by Eq. (5). We fix the BS frequency to $\omega / \mu=0.897$ and vary the coupling constant $\lambda$. In the no-coupling limit, i.e., for $\lambda=0$, the $\omega / \mu=0.897$ mini BS exhibits a $\tilde{m}=2$ NAI with growth rate $M \tilde{\omega}_{I}=1.8 \times 10^{-2}$. As shown in Figure 6 , as $\lambda$ increases, the $\tilde{m}=2$ NAI growth rate decreases (almost linearly), until it approaches zero at

$$
\lambda^{c} / \mu^{2}=133.2 \text {. }
$$

Note, however, that there exists a small interval of $\lambda / \mu^{2}$, for which the NAI is dominated by a $\tilde{m}=1$ mode [which we ignore for the purposes of determining Eq. (16)]. This result seems to indicate that the NAI is turned off for $\lambda / \mu^{2}>133.2$, suggesting that BSs residing in this portion of the parameter space are stable. Finally, in
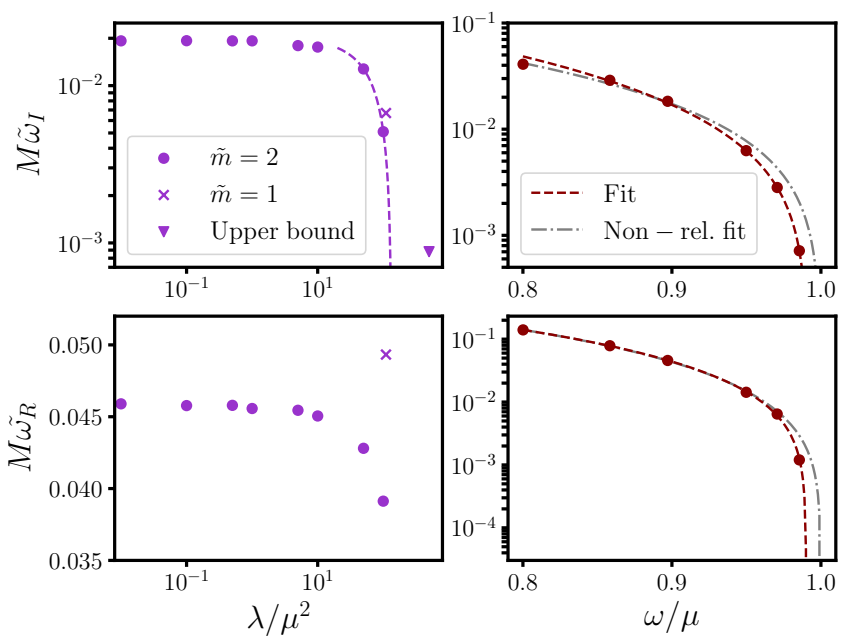

Figure 6. The top and bottom panel on the left show the complex frequency of the unstable modes of a family of repulsive potential BSs with fixed $\omega / \mu=0.897$, but varying coupling constant $\lambda$. In all but one case, the unstable mode has the azimuthal number $\tilde{m}=2$; for $\lambda / \mu^{2}=110$, the $\tilde{m}=1$ mode is the (most) unstable. The dashed curve is a linear interpolation based on the two $\tilde{m}=2$ cases with largest $\lambda / \mu^{2}$ (ignoring the $\tilde{m}=1$ case). The top and bottom panel on the right show the corresponding complex frequencies of the unstable modes of the family of mini BSs. We fit both a generic quadratic ansatz with three degrees of freedom (red dashed) and a quadratic ansatz (gray dash-dotted), which is fixed to $\left.\tilde{\omega}_{R, I}\right|_{\omega=\mu}=0$, to the data. Finally, we indicate the upper bounds on the growth rate of the case considered in Table I by down-arrows.

contrast to the trend in growth rates of the relativistic branch, the real part of the perturbation's frequency $\tilde{\omega}_{R} M$ decreases together with $\tilde{\omega}_{I} M$ to zero at $\lambda^{c} / \mu^{2}$, on this non-relativistic branch. When comparing the importance of the mass term $\mu^{2}|\Phi|^{2}$ to the repulsive potential $\lambda|\Phi|^{4}$ we see that the self-interactions dominate, i.e., $\lambda|\Phi|^{2} / \mu^{2}>1$ (around the maximum of the magnitude of the scalar field inside the star), for $\omega / \mu=0.897$ repulsive BSs with couplings $\lambda / \mu^{2} \geq 100$. This difference grows, with increasing coupling, allowing for the possibility of scalar BSs where the effect of the mass term is small in comparison to the quartic term, except at the outer and inner edges of the star.

In Figure 6, we also show the NAI growth rates of mini BSs in the regime between the global maximum of $\mu M$ and the non-relativistic limit. The growth rates and harmonic frequencies $\tilde{\omega}_{R}$ both decrease approaching $\omega=\mu$. Fitting a generic quadratic ansatz to the $\tilde{\omega}_{I} M$ and $\tilde{\omega}_{R} M$ data, we find the respective critical boson frequencies where these two quantities would go through zero:

$$
\omega_{\mathrm{I}, \mathrm{m}}^{c} / \mu=0.991, \quad \omega_{\mathrm{R}, \mathrm{m}}^{c} / \mu=0.990 .
$$

We perform a resolution study on the mini BS with $\omega / \mu=0.97$ (see Appendix B for details) to determine the numerical uncertainty of our results, and hence, un- 
derstand the stability of BSs with $\omega \approx \mu$. The relative numerical uncertainty for the $\tilde{\omega}_{R, I} M$ estimates is $\approx 3 \%$. Comparing this with the extrapolated critical frequencies Eq. (17), implies that our results are consistent with $\tilde{\omega}_{I}$ and $\tilde{\omega}_{R}$ both reaching zero at $\omega=\mu$. The second fit presented in Figure 6, fixed to obey $\tilde{\omega}_{I}=0$ as $\omega \rightarrow \mu$, is consistent with the free fit to within the numerical uncertainty. These results suggest that the timescales of the NAI grow at least as $\tilde{\tau} / M \sim(1-\omega / \mu)^{-1}$ approaching the non-relativistic limit. Note also, when normalizing the frequencies by the $\mathrm{BS}$ radii, $\tilde{\omega}_{R, I} R_{99}^{K, Q}$, these quantities still tend to zero in the dilute limit (see Sec. III D for a detailed discussion of this). We point out that these results are consistent with Ref. [60]; there a diffuse scalar cloud was evolved to form a rotating BS and then undergo the NAI. They find (notice a factor of two difference in the definition of the $|\Phi|^{4}$-term), a decrease of the instability timescales for larger $\lambda$, which is to be expected based on our results, because (a) the non-relativistic limit is unstable independent of the scalar self-interactions, and (b) the repulsive potential becomes more important the lower $\omega / \mu$. Therefore, the larger $\omega / \mu$, the larger the critical coupling $\lambda^{c} / \mu^{2}$. In fact, one could conjecture: $\lim _{\omega \rightarrow \mu} \lambda^{c} \rightarrow \infty$.

To further test all these results, we pick one BS solution for each potential out of the regime conjectured to be free of the NAI (based on the trends in Figure 5 and Figure 6 ) and evolve it for many dynamical times. To that end, we evolve the cases summarized in Table I up to times $T_{\max }$, while monitoring $\Phi_{m}$ [defined in Eq. (13)] over time. In Sec. III A, we illustrated that tracking $\Phi_{m}$ captures the exponential growth during the linear phase of the NAI. While the truncation error introduces perturbations, these slowly decay away, and - in contrast to Figure 3 - there is no sign of exponential growth for the cases presented in Table I. From this we conclude that any instability develops on significantly longer timescales than the unstable cases with $\omega>\omega^{c}$ and $\lambda>\lambda^{c}$ found above. Based on these simulations, we place upper bounds on the growth rates of possible instabilities arising in those BSs. To that end, we use the unstable cases presented in Figure 5, and extrapolate the exponential growth backwards in time to $t=0$ to estimate the initial amplitude at which truncation error seeds the unstable mode. Assum-

\begin{tabular}{c|cccccc}
\hline \hline$V(|\Phi|)$ & Coupl. & $\omega / \mu$ & $J / M^{2}$ & $C^{K}$ & $C^{Q}$ & $T_{\max } / M$ \\
\hline \hline Axionic & $f=0.005$ & 0.303 & 3.59 & 0.061 & 0.049 & $11.1 \times 10^{3}$ \\
\hline Solitonic & $\sigma=0.05$ & 0.303 & 1.36 & 0.169 & 0.140 & $7.1 \times 10^{3}$ \\
\hline KKLS & $\kappa=0.1$ & 0.382 & 0.91 & 0.333 & 0.290 & $3.7 \times 10^{3}$ \\
\hline Repulsive & $\lambda / \mu^{2}=500$ & 0.897 & 0.64 & 0.077 & 0.078 & $13.5 \times 10^{3}$ \\
\hline \hline
\end{tabular}

Table I. We study the dynamical evolution of a set of $m=1$ BSs, one for each set of scalar self-interactions that is expected to be stable based on the results obtain in Figure 5 and Figure 6. We evolve these cases up to $T_{\max } / M$ and find no sign of an instability arising. ing that this amplitude is similar in the stars considered in Table I, we extract approximate upper bounds on the possible growth rates for an instability not to be evident during the simulated time. We include these in Figure 5 and Figure 6.

Finally, we use nonlinear evolutions to explore the stability of individual $m=2$ rotating BSs in different scalar models, as well as $m=1 \mathrm{BSs}$ in various models with different couplings from the ones shown in Figure 5. The properties of the resulting evolutions are summarized in Table II. In our small set of $m=2 \mathrm{BSs}$, we did not find a solution that does not develop a NAI. Note that, as will be discussed in the next section, the NAI for the $m=2$ BSs has non-negligible support over several azimuthal modes. Therefore, the $\tilde{m}$ values for $m=2 \mathrm{BSs}$ in Table II should be understood as rough estimates.

The result for $m=1$ BSs shown in Table II solidify the conclusions drawn above. We show that even when changing the coupling constants in scalar models considered in Figure 5, there is an unstable (high frequency) regime, but also a seemingly stable (low frequency) regime, both in the solitonic and the axionic model. This shows that the coupling parameters chosen in Figure 5 are not special. In addition to this, we also study the stability of BSs in scalar models with a Liouville potential [66] [defined in Eq. (6)], a non-perturbative extension of the repulsive self-interactions. Analogous to the family of BSs in the repulsive model, studied in Figure 6 , we fix the BS frequency to $\omega / \mu=0.9$ and vary the coupling $\alpha$. As seen from Table II, there is a low-coupling range, for which an $\tilde{m}=2 \mathrm{NAI}$ is present, but also a large coupling regime, where the NAI is quenched, and no sign of an instability can be found. Therefore, the addition of a $|\Phi|^{4}$ term in the scalar potential, is sufficient to stabilize rotating BS in a portion of the parameter space, and

\begin{tabular}{c|cccccc}
\hline \hline$V(|\Phi|)$ & Coupl. & $m$ & $\omega / \mu$ & $C^{K}$ & $\tilde{m}$ & $M \tilde{\omega}_{I}$ \\
\hline \hline KKLS & $\kappa=0.1$ & 2 & 0.6 & 0.26 & 4 & $7.4 \times 10^{-2}$ \\
\hline Axionic & $f=0.1$ & 2 & 0.9 & 0.08 & 2 & $1.1 \times 10^{-2}$ \\
\hline Solitonic & $\sigma=0.05$ & 2 & 0.4 & 0.14 & 2 & $5.3 \times 10^{-2}$ \\
\hline Solitonic & $\sigma=0.05$ & 2 & 0.9 & 0.03 & 4 & $8.5 \times 10^{-2}$ \\
\hline \hline Solitonic & $\sigma=0.1$ & 1 & 0.8 & 0.08 & 2 & $1.3 \times 10^{-1}$ \\
\hline Solitonic & $\sigma=0.1$ & 1 & 0.4 & 0.28 & - & $<5.4 \times 10^{-3}$ \\
\hline Axionic & $f=0.01$ & 1 & 0.8 & 0.03 & 2 & $4.5 \times 10^{-2}$ \\
\hline Axionic & $f=0.01$ & 1 & 0.3 & 0.19 & - & $<5.3 \times 10^{-3}$ \\
\hline Liouville & $\alpha=0.1$ & 1 & 0.9 & 0.07 & 2 & $1.0 \times 10^{-2}$ \\
\hline Liouville & $\alpha=0.05$ & 1 & 0.9 & 0.07 & - & $<2.8 \times 10^{-3}$ \\
\hline \hline
\end{tabular}

Table II. Properties of several additional rotating BSs are considered and their dynamical behavior studied. $\tilde{m}$ refers to the azimuthal number of the dominant (i.e., most unstable) nonaxisymmetric mode. However, as pointed out in the text, for $m=2 \mathrm{BSs}$, the NAI is composed of several competing modes simultaneously. We estimate upper bounds on the growth rates for the cases, where no sign of an instability can be found. We describe in the text how these upper bounds are obtained. 
a)

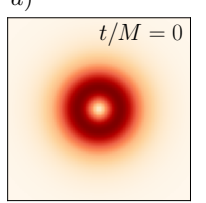

b)

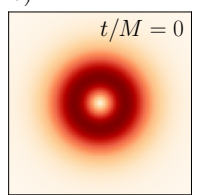

c)

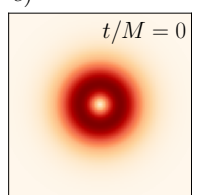

d)

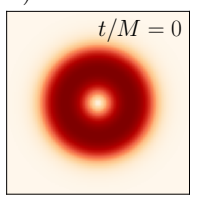

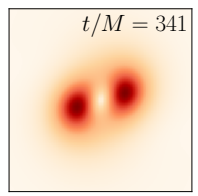
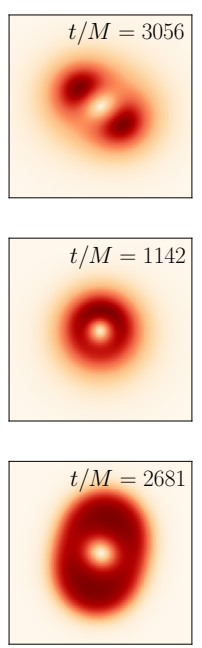
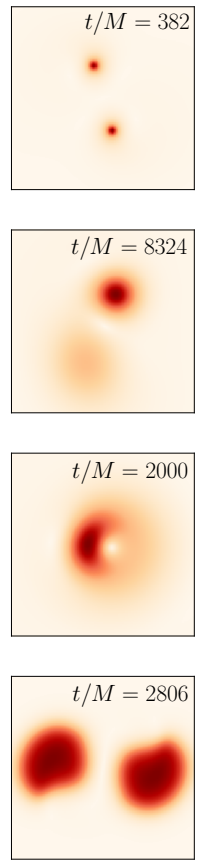

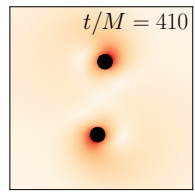

$t / M=9806$
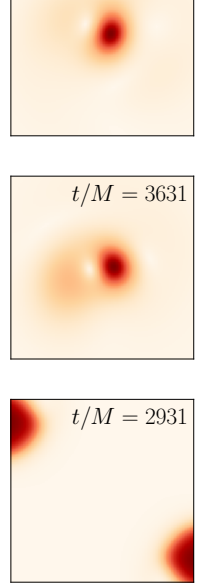

Figure 7. Series of snapshots (increasing in time from left to right) showing the evolution of $|\Phi|^{2}$ in four different scenarios where a BS undergoes a NAI. These are representative of the possible end states of all the unstable $m=1 \mathrm{BSs}$ studied here. From top to bottom: a) Evolution of an $m=1$ mini BS with $\omega / \mu=0.8$ that collapses to a binary $\mathrm{BH}$ (the regions inside the apparent horizons are indicated in black). b) Snapshots of an $\omega / \mu=0.95 m=1$ mini BS resulting in a non-rotating BS with non-negligible linear momentum. $c$ ) The NAI of an $m=1$ rotating BS, in the repulsive model with $\lambda / \mu^{2}=110$, and $\omega / \mu=0.897$ (corresponding to the $\tilde{m}=1$ case in Figure $6)$, yielding a non-rotating BS with large linear momentum. d) Finally, the evolution of an $m=1$ axionic BS with $\omega / \mu=$ 0.425 where the NAI results in the fragmentation of the star into two equal-mass non-rotating BSs.

a potential that has additional higher order terms does not affect this qualitative behavior.

\section{Results: Endstate of the NAI}

In the previous section, we identified two distinct regimes, the relativistic and non-relativistic branch, where the NAI is quenched and the stars appear stable when evolved nonlinearly. In what follows, we explore the end state of the instability of $m=1$ and $m=2 \mathrm{BSs}$ both in the relativistic regime and the non-relativistic regime.

In Ref. [43], it was found that a $m=1$ mini BS with $\omega / \mu=0.83$ was subject to a $\tilde{m}=2$ NAI that lead to the formation of a $\mathrm{BH}$. We study this family of mini BSs in more detail, following the growth and end state of the instability for the cases shown in Figure 6 with $\omega / \mu \in\{0.80,0.86,0.90,0.95\}$. These stars undergo a

a)

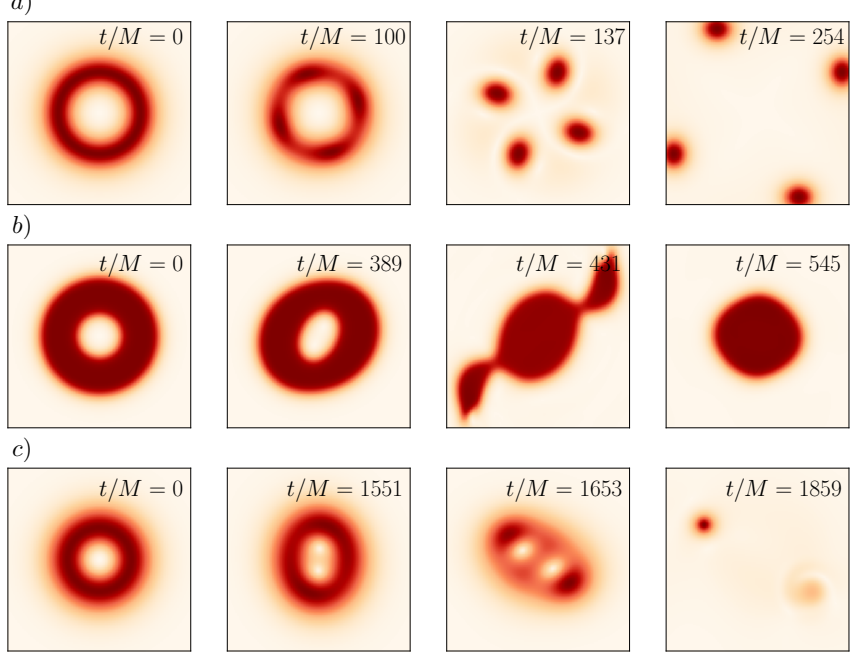

Figure 8. Analogous to Figure 7, here we present the dynamics of three $m=2$ BSs undergoing a NAI (their properties can be found in Table II). From top to bottom: a) The evolution of a rotating BS, in the KKLS model with $\kappa=0.1$, and $\omega / \mu=0.6$, results in four non-rotating equal mass BSs that are flung out from the center of mass. $b$ ) The fragmentation of a solitonic BS with $\sigma=0.05$ and $\omega / \mu=0.4$ into a large, oscillating, approximately spherically symmetric BS at the center, and two non-rotating BSs flung out at relativistic velocities. c) Finally, the NAI of an axionic BS with $f=0.1$ and $\omega / \mu=0.9$, undergoing a complex fragmentation process resulting in a single non-rotating BS with significant linear momentum.

$\tilde{m}=2$ NAI, form two approximately non-rotating BSs that orbit around each other, and collapse to a binary $\mathrm{BH}$ (for $\omega / \mu \in\{0.80,0.86\}$; see panel $a$ ) in Figure 7 ), or merge into a single non-rotating $\mathrm{BS}$ that then collapses to an isolated $\mathrm{BH}$ (for $\omega / \mu=0.90$ ). In the case of $\omega / \mu=0.80 \mathrm{mini} \mathrm{BSs}$, the individual BSs collapse to $\mathrm{BHs}$ with negligible spin, and subsequently merge into a single $\mathrm{BH} \approx 60 M$ later. Additionally, we find that mini BSs with $\omega / \mu=0.95$ undergo an $\tilde{m}=2$ NAI and settle to a (highly perturbed) non-rotating BS (that does not collapse further), through the emission of scalar and gravitational waves, with non-zero linear momentum (see panel $b$ ) in Figure 7). This suggests that the contributions from the $\tilde{m}=1$ unstable mode is non-negligible in this case. Similarly, repulsive self-interactions Eq. (5) with sufficiently large coupling can prevent the BSs from collapsing to BHs. Instead, the $m=1 \mathrm{BSs}$ in the repulsive model, considered in Figure 6, undergoing the $\tilde{m}=2$ NAI, collapse to BHs for couplings up to $\lambda / \mu^{2}=50$. For $\lambda / \mu^{2} \geq 100$, however, we find that the $\tilde{m}=2$ NAI eventually results in an approximately spherically symmetric BS, while the instability is quenched at Eq. (16). In Figure 7 , we also depict the nonlinear evolution of a BS undergoing a $\tilde{m}=1$ NAI.

This results in a highly perturbed, approximately nonrotating $\mathrm{BS}$, with significant linear momentum, radiating both scalar and gravitational waves outwards. We 
can conclude that, the closer the BS is to the critical frequency/coupling, the less it seems to be prone to forming a BH. Therefore, we can also conclude that the end state of the NAI for $m=1 \mathrm{BSs}$ on the branch directly connected to the non-relativistic limit depends both on the nonlinear scalar self-interactions, as well as the BS frequency. In contrast to this, we find a consistent outcome for the final fate of $m=1 \mathrm{BSs}$ undergoing the NAI on the relativistic branch. All BSs presented in Figure 5 have the same final state, independent of the potential or their frequency: after the $\tilde{m}=2 \mathrm{NAI}$, two approximately non-rotating BSs are formed that are flung out into opposite directions at relativistic speeds. This behavior is illustrated in panel $d$ ) of Figure 7 .

We can conclude that the end state of the NAI in $m=1 \mathrm{BSs}$ is clearly differentiated in the two branches. The first non-relativistic branch exhibits quasi stable bound non-stationary states after fragmentation of the original $\mathrm{BS}$ that either settle into a $\mathrm{BH}$ or to a single non-rotating BS. The second, relativistic branch consistently results in two non-rotating BSs that are flung out after fragmentation, independent of the frequency or the character of the nonlinear scalar self-interactions.

In Figure 8, we show the evolution of $m=2$ BSs undergoing a NAI. The dynamics of the NAI in these stars is more complex, as more unstable non-axisymmetric modes are of non-negligible size. While the linear phase is still mostly dominated by a single azimuthal mode $\tilde{m}$ (see also Table II), the nonlinear evolution of these $m=2$ BSs is substantially different from their $m=1$ counterparts.

\section{Results: Physical origin of the NAI}

In this section, we investigate the possible physical mechanisms leading to stable regions in the BS parameter space. We focus on the case of $m=1 \mathrm{BSs}$ and distinguish between stars on the relativistic branch and the non-relativistic branch, as the NAI of each has fundamentally different properties.

First, let us consider the non-relativistic branch, i.e., mini BSs and stars in the repulsive scalar model with frequencies between the non-relativistic limit $\omega / \mu=1$ and the global maximum of the BS's mass. Since the solitonic, axionic, and KKLS scalar models reduce to the mass term in the Newtonian limit (for those cases, the non-relativistic branch extends from $\omega / \mu=1$ to the local maximum of the BS's mass), the following also applies to that part of the BS parameter space in those models. From the previous section, we recall that the NAI timescales of mini BSs tend to infinity in the Newtonian limit, while BSs in the repulsive scalar model with fixed frequency $\omega / \mu=0.897$ turn stable for coupling parameters with $\lambda>\lambda^{c}=133.2 \mu^{2}$. In Ref. [60], it was suggested that the presence of a co-rotation point (the radius at which angular velocity of the matter and the pattern speed of the unstable modes are equal) inside the star,

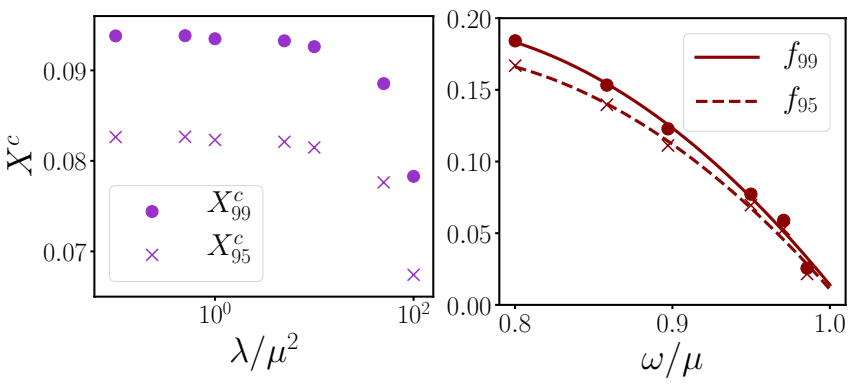

Figure 9. We plot the normalized difference $X_{i}^{c}=\left(R_{i}^{K}-\right.$ $\left.R_{\text {cor }}\right) / R_{i}^{K}, i \in\{99,95\}$, between the co-rotation point $R_{\text {cor }}$ and the two radii $R_{99}^{K}$ and $R_{95}^{K}$ for the families of BSs considered in Figure 6. The left panel shows the repulsive potential cases, and the right shows the mini BS cases. The quantity $R_{95}^{K}$ is defined, analogously to how $R_{99}^{K}$ is in Sec. II B, as the circular radius $r=R_{95}^{K}$, where $95 \%$ of the energy resides in $r<R_{95}^{K}$. In the right panel, the solid and dashed lines are quadratic fits to the $X_{99}^{c}$ and $X_{95}^{c}$ data points.

is driving the NAI. There it was conjectured that, if the co-rotation point is outside the star (or it does not exist at all), then the star is stable, while if such a point exists within the star's radius, the star is unstable. Here we find this conjecture to hold for BSs on the non-relativistic branch. (Though not for the relativistic branch, as we shall discuss below.) The angular velocity $\tilde{\Omega}$ is defined in terms of the energy density $\rho$ and angular momentum density $J^{\mu}$, with respect to slices of constant time with unit normal $n^{\mu}$ and projector $\gamma_{\mu \nu}=g_{\mu \nu}+n_{\mu} n_{\nu}$ :

$$
\tilde{\Omega}=J^{\varphi} / \rho, \text { with } \quad J_{i}=\gamma_{i}^{\mu} n^{\nu} T_{\mu \nu}, \quad \rho=n^{\mu} n^{\nu} T_{\mu \nu} .
$$

(Note that we are only measuring this quantity with respect to our stationary and axisymmetric solutions.) We define the co-rotation radius to be the radius $r=R_{\text {cor }}$ in the equatorial plane of a rotating BS where $\tilde{\Omega}\left(R_{\text {cor }}\right)=$ $\tilde{\omega}_{R} / \tilde{m}$.

In Figure 9, we show that $R_{\text {cor }}$ approaches the radius of mini BSs, as well as BSs in the repulsive scalar model, as those families approach the critical frequency $\omega^{c}$ and critical coupling $\lambda^{c}$, respectively. This holds for either definition of the radius of the BS. In the repulsive scalar model, this is not surprising, since we already saw in Sec. III B that the real part of the unstable mode's frequency (and therefore, the pattern speed $\tilde{\omega}_{R} / \tilde{m}$ ), tends to zero at $\lambda^{c}$. While the radius remains finite passing through $\lambda^{c}$, the decreasing pattern speed is pushing $R_{\text {cor }} \rightarrow \infty$, as $\lambda \rightarrow \lambda^{c}$. In the mini BS case, however, this is not the case. In the Newtonian limit, $\omega / \mu \rightarrow 1$, the radius of the BS diverges $R_{99}^{K} \rightarrow \infty$, while simultaneously, $\tilde{\omega}_{R} \rightarrow 0$. Therefore, there are two competing effects determining, whether $R_{99}^{K}<R_{\text {cor }}$ or $R_{99}^{K}>R_{\text {cor }}$. Despite this, the co-rotation point approaches the radius of the BS from inside the star, while the NAI growth rate decreases, as can be deduced from Figure 9. On top of this, we explicitly checked that $\lim _{\omega \rightarrow \mu}\left(\tilde{\omega}_{R, I} R_{99}^{K}\right)=0$. This suggests that the presence of the co-rotation radius 

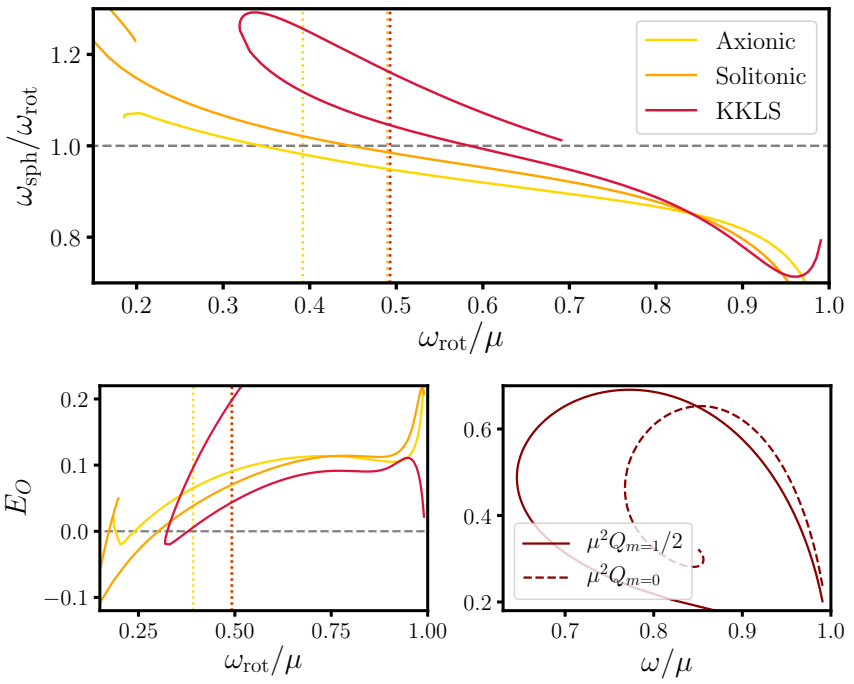

Figure 10. Top panel: The ratio $\omega_{\mathrm{sph}} / \omega_{\text {rot }}$ of the boson frequency in $m=1 \mathrm{BSs}$ with charge $Q$ to the frequency of $m=0$ BSs with charge $Q / 2$ in several scalar models. The ratios pass through unity at $\omega_{A}^{c} / \mu=0.34, \omega_{S}^{c} / \mu=0.45$ and $\omega_{K}^{c} / \mu=0.58$ for the axionic, solitonic and KKLS models, respectively. For comparison, we indicate the critical frequencies, in Eq. (15), by dashed vertical lines. Bottom left: The orbital energy $E_{O}$, defined in Eq. (19), of a $m=0$ binary BS system with constituent charge $Q / 2$ emerging from a single $m=1 \mathrm{BS}$ with charge $Q$. (Legend from top panel also applies here.) Bottom right: Half the scalar charge $Q / 2$ for $m=1 \mathrm{mini}$ BSs (solid line), compared with the full charge $Q$ of $m=0$ mini BSs (dashed line), as a function of their respective frequencies.

inside the star (independent of the notion of radius) is related to the instability of $\mathrm{BSs}$ on the non-relativistic branch. Lastly, this also seem to indicate that there is no finite regime with stable mini BSs in the Newtonian limit between the values in Eq. (17) and $\omega / \mu=1$.

Let us now discuss the relativistic branch, i.e., axionic, solitonic, and KKLS BSs with frequencies between the local and the global maximum of $M$ (see Figure 2): $0.3 \lesssim \omega / \mu \lesssim 0.9$. Recall from the discussion in Sec. III C that these families of BSs undergo a $\tilde{m}=2$ NAI that results in two equal-mass non-rotating BSs being flung out from the center of mass, for initial BS frequencies $\omega^{c}<\omega \lesssim 0.9 \mu$. Fundamentally, the system posses three distinct conserved quantities: the ADM mass $M$, the angular momentum $J$ about the symmetry axis, and the $\mathrm{U}(1)$-charge $Q$. Given that the total number of bosons is conserved, we neglect scalar radiation and assume that the final state of the instability of a $m=1 \mathrm{BS}$ with charge $Q_{\text {rot }}$ is a binary $m=0$ BS of total charge $2 Q_{\mathrm{sph}}=Q_{\mathrm{rot}}$. From the difference in the initial BS mass, and that of the putative binary, we can calculate the normalized orbital energy

$$
E_{O}=\left(M_{\text {rot }}-2 M_{\mathrm{sph}}\right) / M_{\mathrm{rot}},
$$

as well as the frequency $\omega_{\text {sph }}$ of BSs in the binary, as a function of initial boson frequency $\omega_{\text {rot }}$. We show this in Figure 10. Based on the values shown there, the NAI fragments should be significantly unbound: $E_{O}=0.1$ corresponds to a velocity of $\approx 0.4 c$ at infinity. All the unstable solutions on the relativistic branch that we find occur in the regime where $E_{O}>0$, which is consistent with the fact that the instability gives rise to two fragments which appear unbound. However, there is some part of the parameter space where $E_{O}>0$, but we do not find the NAI.

The critical frequencies in Eq. (15), where the NAI instability approaches zero, seem to roughly align with those, where $\omega_{\text {sph }}=\omega_{\text {rot }}$ (see Figure 10). Due to the fragmentation, and ignoring radiation, the bosons can fall into lower frequency/energy states within the stars (since there $\omega_{\text {sph }}<\omega_{\text {rot }}$ ), while for the regime where $\omega_{\text {sph }}>\omega_{\text {rot }}$, the bosons would have to climb up into higher frequency states, if the rotating BSs were to fragment into two non-rotating BSs. Hence, one can conjecture that the points, where $\omega_{\text {sph }}=\omega_{\text {rot }}$, indicate the switching of the stability properties. However, there is a discrepancy between the critical frequencies where $\omega_{\text {sph }}=\omega_{\text {rot }}$ in Figure 10, and those derived from the exponentially growing modes in Eq. (15) (even when including the numerical uncertainty), indicating that this criterion based on the relative frequency of the rotating and non-rotating equilibrium BS solutions is at most approximate. Furthermore, this approximate argument cannot be applied in the same way to the non-relativistic branch. Recall from Sec. III C that the NAI in the case of mini BSs yields two orbiting pieces that either collapse to $\mathrm{BHs}$ or form a single non-rotating BS. Assuming these two orbiting scalar field distributions are roughly stationary isolated spherically symmetric BSs, i.e., applying the above argument, and consulting Figure 10 (bottom right), we notice two features. One is that $\omega_{\text {rot }}<\omega_{\text {sph }}$ for most of the parameter space, at least when comparing the turning-point stable branches. This may be related to the fact that the BS fragments collapse in some cases, which would be consistent with them being unstable spherical stars. The second feature to note is that for $m=1$ stars with $\omega_{\text {rot }} / \mu>0.846$, there exists no corresponding non-rotating counterparts with half the U(1)charge. Again, this may be related to the fact that for large enough $\omega_{\text {rot }}$, the instability gives rise to a single $\mathrm{BH}$ or non-rotating BS. Additionally, we observe no significant qualitative change in the dynamics of the systems when moving across the point where both curves cross.

Finally, we note that the co-rotation argument discussed above in the context of non-relativistic BSs does not apply to the relativistic branch. This is because the real part of the unstable mode's frequency remain non-zero on the entire relativistic branch (see Figure 5), and hence the co-rotation point remains well inside the star for all unstable BSs. Even when extrapolating $\tilde{\omega}_{R} M$ deeper into the relativistic regime, the co-rotation point seems to only exit the star at the global maximum of the BS's mass. 


\section{DISCUSSION AND CONCLUSION}

In this work, we study the stability of $m=1$ and 2 $\mathrm{BSs}$ in various massive complex scalar models minimally coupled to gravity. We do this by numerically evolving the Einstein-Klein-Gordon system of equations, starting from stationary BS initial data. We consider a number of different types of scalar interactions, and explore the parameter space of BS solutions. We find that all $m=1$ mini BSs are unstable to an exponentially-growing, nonaxisymmetric mode with azimuthal number $\tilde{m}=2$, but that the growth rate scales as $\tilde{\omega}_{I} \propto(1-\omega / \mu)$ when $\omega \rightarrow \mu$ (see Figure 6). Since all nonlinear scalar self-interactions reduce to the mass term for sufficiently small field values, this holds also for all $m=1$ scalar BS that reside sufficiently deep in the Newtonian limit. This may have some relevance for rotating distributions of dark matter in models where they are composed of ultralight bosons.

We find that adding a nonlinear scalar potential can quench the instability in a number of cases, in the sense that the growth rate approaches zero as the BS frequency decreases towards a critical value (see Figure 5 and Figure 6). Studying the conjecture in Ref. [60], we relate the stability of the stars on the non-relativistic branch to the presence of the co-rotation point inside the star, and show that, when the BSs become stable, the corotation point moves outside of the star (independent of the notion of radius; see Figure 9). The stability of solutions on the relativistic branch seems unrelated to the co-rotation, though approximately related to the BS frequency; the final state of the instability is always an equal-mass binary BS of non-rotating constituents, and we find around where the rotating stars become stable, there is a transition from this final state having lower frequency to having higher frequency (see Figure 10 for the details). It would be interesting for future work to see if this argument could be sharpened. In Refs. [43, 60], it was argued that the stability of BSs is related to the morphology of surfaces of constant scalar energy. Stars with toroidal morphology were claimed to be unstable, while those with spherical surfaces of constant energy, stable. Here we find $m=1$ scalar BSs that have toroidal morphology, but do not appear to be subject to this instability. Though we do not explore the parameter space of $m=2 \mathrm{BSs}$ as thoroughly as the $m=1$ case, we find all the examples we consider to be linearly unstable.

We choose several examples of $m=1$ BSs with nonlinear interactions that are past the point where the NAI growth rate approaches zero, and evolve them for many dynamical times (in some cases $>10^{4} M$ ) and find no sign of a growing perturbation. These include cases with high compactness, rapid rotation, and -in the case of the KKLS BS in Table I-unstable and stable photon orbits, which can be used for future studies of mergers, and compared to the mergers of black holes and neutron stars. Of course, our methods can not definitely rule out instabilities of any kind, in particular instabilities that arise on longer timescales than considered here. For ex- ample, in Ref. [83], it was conjectured that stable photon orbits may give rise to nonlinear instability, based on the slow timescales associated with the decay of linear perturbations. If such a nonlinear instability were to exist, it would likely operate on much longer timescales than the NAI, and so may not be evident here.

Another future direction is to see whether rotating scalar BSs could be formed dynamically, through a nonspinning binary BS merger, or through the collapse of a rotating scalar cloud. The parameter space identified here could be used to choose favorable initial conditions that may dynamically give rise to a stable rotating $\mathrm{BS}$. It would also be interesting to investigate scenarios where unstable rotating BS solutions may form, for example from the migration of a stable star to a unstable part of the parameter space, or through rapid collapse. In such cases, the nonlinear development of the instability will give rise to distinct gravitational wave signatures. For example, here we find cases where the NAI of a rotating BS gives rise to a binary $\mathrm{BH}$ which will subsequently merge, similar to the binary $\mathrm{BH}$ formation found to arise in the collapse of supermassive stars [84].

\section{ACKNOWLEDGMENTS}

We acknowledge support from an NSERC Discovery grant. Research at Perimeter Institute is supported in part by the Government of Canada through the Department of Innovation, Science and Economic Development Canada and by the Province of Ontario through the Ministry of Economic Development, Job Creation and Trade. This research was enabled in part by support provided by SciNet (www.scinethpc.ca) and Compute Canada (www.computecanada.ca). Calculations were performed on the Symmetry cluster at Perimeter Institute and the Niagara cluster at the University of Toronto.

\section{Appendix A: Numerical construction of stationary boson stars}

Here we provide the details of the numerical methods employed in order to construct stationary BS solutions. We assume an axisymmetric and stationary metric $g_{\mu \nu}$, which can be written in Lewis-Papapetrou coordinates as in Eq. (7), where all free functions depend only on the $r$ and $\theta$ coordinates. Additionally, we use a harmonic scalar field ansatz $\Phi=e^{i \omega t+i m \varphi} \phi$, where $\phi$ carries the $r$ and $\theta$ dependence, that is compatible with the metric ansatz. Plugging these expressions into the EinsteinKlein-Gordon equations, Eq. (8), results in a set of five coupled partial differential equations in $r$ and $\theta$. Imposing regularity at the origin, $r=0$, and asymptotic flatness at $r \rightarrow \infty$ provides the boundary conditions for the elliptic set of equations. See, for instance, Ref. [62] for the explicit form of the equations and boundary conditions for the metric ansatz Eq. (7) chosen here. Notice a 
typo in their Eq. (B1): The second to last term should be $-2 l\left(\partial_{r} f\right)^{2}$. Additionally, we find our axisymmetric system of equations to agree if, in the equation for $f(r, \theta)$ (i.e., Eq. (B6) in Ref. [62]):

$$
\begin{aligned}
-\frac{1}{2} \partial_{\theta} f & \rightarrow-\frac{1}{2} \frac{\partial_{\theta} f \partial_{\theta} l}{l}, \\
-2 r^{2} \kappa l U(\phi) & \rightarrow-2 r^{2} \kappa l g U(\phi) .
\end{aligned}
$$

As mentioned in the main text, we introduce the auxiliary functions $\omega_{s}$ and $\rho$. Following the arguments in Ref. [62], these obey the equations

$$
\Delta_{g} \omega_{s}=0, \quad \Delta_{g} \rho=j^{t} / \omega_{s},
$$

where $\Delta_{g}:=g^{i j} \nabla_{i} \nabla_{j}$ is the Laplacian associated with the spacetime metric $g_{\mu \nu}$, and $j^{t}$ is proportional to the charge density defined by the Noether current associated with the global U(1) symmetry. Here we have also promoted the scalar field's frequency $\omega$ to the scalar function $\omega_{s}$, which, however, is fixed to be constant by the Laplace equation (with the appropriate boundary conditions given below). Introducing $\omega_{s}$ and $\rho$ in such a fashion enables us to impose either the BS's frequency $\omega$, or the total $\mathrm{U}(1)$-Noether charge $Q$, as boundary conditions at spatial infinity (outlined below). This way, we are able to explore the full parameter space, even, when the family of solutions develops two different branches for a fixed frequency $\omega$ or charge $Q$.

We solve these equations using a relaxation method, which requires a sufficiently good guess for the field configurations. In order to obtain such an initial guess, we begin by exploiting the fact that Eq. (3) admits solitonic solutions even in the absence of gravity. In the nonrotating limit, the above set of elliptic partial different equations reduces to a single ordinary differential equation for $\phi(r)$. We compactify the radial coordinate to $\bar{r} \in(0,1)$ with $\bar{r}=r /(1+r)$. Given the boundary conditions for $\phi$, we use a shooting method, starting at the origin, and integrating outwards to $\bar{r}=1$, to generate the non-gravitating scalar solitons (known as Q-balls) in the non-relativistic regime (i.e., where $\omega / \mu \approx 1$ ). We use these Q-ball solutions as the starting point for solving the equations including gravity. In anticipation of the rotating case, we use a Newton-Raphson-type relaxation code with fifth order finite differences to solve for these spherically symmetric BSs. In order to be able to impose either the BS frequency $\omega / \mu$, or its $\mathrm{U}(1)$-charge $Q$ at $r \rightarrow \infty$, we introduce the two equations Eq. (A3) into the relaxation scheme. The boundary conditions for these auxiliary functions are

$$
\left.\partial_{r} \rho\right|_{r=0}=0, \quad \quad \quad \lim _{r \rightarrow \infty} \rho=\rho_{\infty},
$$

where $\rho_{\infty}$ is arbitrary (we set $\rho_{\infty}=1$ ). We can then impose the BS frequency $\omega$ as a boundary condition given

$$
\lim _{r \rightarrow \infty} \omega_{s}=\omega
$$

or the $\mathrm{U}(1)$-charge by

$$
\lim _{r \rightarrow \infty} 8 \pi r^{2} \omega_{s} \partial_{r} \rho=Q
$$

This follows directly from a volume integration of the $\rho$-equation in Eq. (A3). Finally, in both cases $\lim _{r \rightarrow 0} \partial_{r} \omega_{s}=0$. Gravity can be incorporated slowly starting from the Q-ball solutions in the non-relativistic limit by increasing $\kappa$ from 0 incrementally. Depending on the convergence properties of the individual solutions in the relaxation scheme, the step size $\delta \kappa$ must be adjusted. We find that $\delta \kappa=0.01$ is typically sufficient.

Using these non-rotating BS solutions in the nonrelativistic limit as a starting point, the rest of the parameter space can be explored by marching iteratively in the $\omega / \mu$ direction: We start from a solution with $\omega=\omega_{0}$, take a sufficiently small step $\omega_{1}=\omega_{0}-\delta \omega($ with $\delta \omega>0)$ towards small frequencies, use the solution with $\omega_{0}$ as an initial guess, and then relax using the above established relaxation scheme into the new solution imposing $\omega=\omega_{1}$. Due to the spiral feature of the solutions in the $(\omega, Q)$ plane, we can follow this approach only up to a global minimum, $\omega_{\text {min }}$, at which point no new solutions can be found at $\omega<\omega_{\min }$. At these turning points, we switch from imposing the frequency $\omega_{\infty}$, to imposing the corresponding BS U(1)-charge with Eq. (A6). We then proceed by incrementally decreasing (or increasing) the charge until we reach the next turning point, where we switch back to imposing the frequency with Eq. (A5), until the family of BS solutions is generated across the parameter space. Spherically symmetric BSs with different potentials can be generated using this family of KKLS BSs as an initial seed for the relaxation into the BS solution with a different potential in the non-relativistic limit (for a given $\kappa$ ). We find this works, even if the two potentials cannot be continuously deformed into each other, as long as the coupling parameters are chosen such that the potentials are similar.

For rotating BSs, we proceed in much the same fashion. In this case, however, we cannot obtain the initial rotating Q-balls using a shooting method, as the equation is no longer an ordinary differential equation, but a partial differential equation in $r$ and $\theta$. We found that generating analytic seeds based on the results of Ref. [62] for $m>0$ rotating Q-balls provided sufficiently good initial guesses to be able to relax into the correct rotating Qball solutions in the non-relativistic regime. Once these solutions are generated, we follow the same procedure as in the $m=0$ case. We find that the necessary resolution varies significantly with the properties of the BS considered. For less compact BSs, $C<0.2$, a resolution of $N_{\bar{r}} \times N_{\theta}=350 \times 50$ (or even lower resolution) was sufficient, while for BSs with high compactness, resolutions up to $500 \times 100$ were necessary for a successful relaxation. In general, we find a resolution of $N_{\bar{r}} \times N_{\theta}=500 \times 200$ is sufficient to generate initial data that accurate enough so that the evolution errors are dominant, even for the highest resolution simulations we consider. 

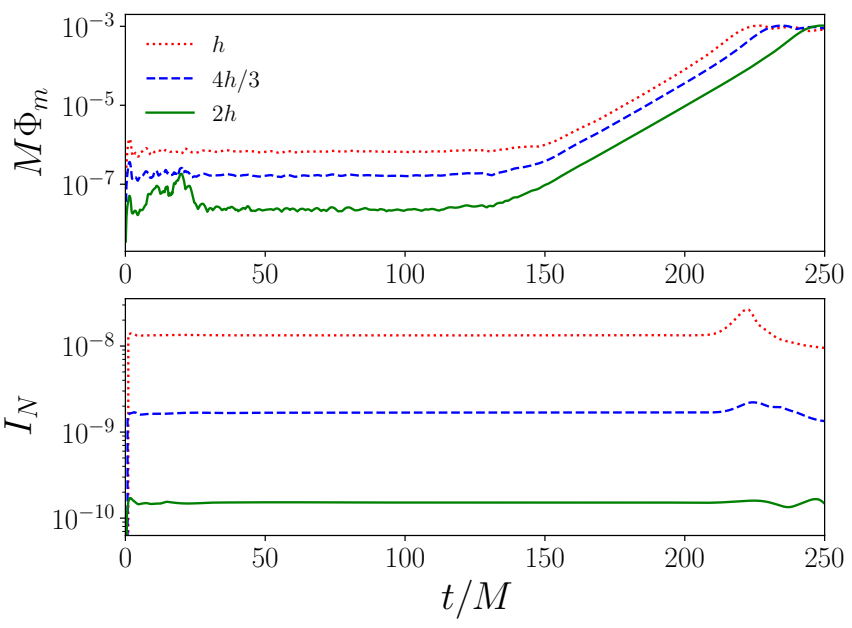

Figure 11. Time evolution of $\Phi_{m}$ (top panel), defined in Eq. (13), at the three different resolutions specified in the text. We also show the integrated norm of the generalized harmonic constraint violation $I_{N}:=\int_{\Sigma_{t}} d^{3} \sigma\left|H_{\mu}-\square x_{\mu}\right|$ (bottom panel). Both quantities converge to zero at the expected fourth order. The origin of the perturbations of $\Phi_{m}$ at early times is discussed in the text. The units for $I_{N}$ are arbitrary since here our purpose is just to demonstrate convergence.

\section{Appendix B: Numerical evolution of boson stars}

Once the stationary solutions are generated, we evolve the Einstein-Klein-Gordon equations in the generalized harmonic formulation [85]. We utilize standard fourth order accurate finite difference stencils and fourth order Runge-Kutta time integration, and use adaptive mesh refinement (AMR). Our computational grid extends to spatial infinity, where we apply boundary conditions that the metric is flat and the scalar field is zero, through the use of compactified coordinates. More details can be found in Ref. [86]. We use a gauge where we fix the source functions $H^{a}$ to be equal to those calculated from the stationary solution, and constant in time. For those mini BSs that collapse to binary (or single) BHs, we utilize a damped harmonic gauge $[53,87]$. We consider a range of cases where the BS compactness varies significantly from $\sim 10^{-3}$ to $\approx 1 / 3$. Therefore, we employ grid hierarchies with between five and eight levels of mesh refinement (using a 2:1 refinement ratio) centered on the BS.

We perform resolution studies, using three different resolutions, on $m=1$ axionic and KKLS BSs with $\omega / \mu=0.6$ (and $M / R_{99}^{K}=0.02$ ) and $\omega / \mu=0.7$ (and $\left.M / R_{99}^{K}=0.14\right)$, respectively, as well as a mini BS with $\omega / \mu=0.97$, to check convergence and determine the numerical accuracy of our results. In Figure 11, we present the time evolution of the magnitude of the scalar field, captured by Eq. (13), and the integrated norm of the constraint violations of the KKLS BS case. For this case, the lowest resolution is $49^{3}$ with six levels of mesh refinement and a spatial resolution of $d x / M \approx 0.26$ on the lowest level. The medium and high resolutions are, respectively, a factor of $4 / 3$ and $2 \times$ larger than the lowest resolution. Both quantities are converging to zero, consistent with the fact that the instability is seeded only by truncation error. The resolution studies for the other cases showed similar behavior. We note that there is transient perturbation to the global maximum $\Phi_{m}$ that is briefly evident in the highest resolution of Fig. 11 that is not converging at the expected rate. This is due to the time interpolation on the AMR boundaries, and in particular an inaccuracy in how the "past" time level used to perform this interpolation at the initial time is set.

The medium resolution used in the resolution studies is equivalent to the resolution we use for all the other cases studied here. We place the mesh refinement such that both radii of the $\mathrm{BS}$ (defined in Sec. II B) reside inside the finest AMR level, and set the spatial resolution to be roughly $d x / R_{99}^{K} \approx 0.04$ or greater in all cases considered in the text. For most cases, we fix the mesh refinement to be that of the initial time slice. In those cases where the NAI yields binary BHs or binary BSs, however, we employ AMR set by the truncation error estimates between different refinement levels to track the fragments. Using Richardson extrapolation, we are able to determine the numerical accuracy of the NAI growth rate and harmonic frequency estimates both on the relativistic and the non-relativistic branch of BS solutions. Based on the respective resolution studies of the $m=1$ KKLS, axionic, and mini BSs, we estimate the relative numerical error in $\tilde{\omega}_{I}$ to typically be $2.5 \%(3 \%)$ on the relativistic (non-relativistic) branch. The error in $\tilde{\omega}_{R}$ is smaller; the relative numerical error in this quantity is $<0.5 \%(3 \%)$ on the relativistic (non-relativistic) branch.
[1] B. P. Abbott et al. (LIGO Scientific, Virgo), Phys. Rev. Lett. 116, 061102 (2016), arXiv:1602.03837 [gr-qc].

[2] B. Abbott et al. (LIGO Scientific, Virgo), Phys. Rev. X 9, 031040 (2019), arXiv:1811.12907 [astro-ph.HE].

[3] R. Abbott et al. (LIGO Scientific, Virgo), (2020), arXiv:2010.14527 [gr-qc].

[4] R. Genzel, A. Eckart, T. Ott, and F. Eisenhauer, MNRAS 291, 219 (1997).

[5] A. Ghez, B. Klein, M. Morris, and E. Becklin, Astrophys.
J. 509, 678 (1998), arXiv:astro-ph/9807210.

[6] K. Akiyama et al. (Event Horizon Telescope), Astrophys. J. 875, L1 (2019), arXiv:1906.11238 [astro-ph.GA].

[7] V. Cardoso and P. Pani, Living Rev. Rel. 22, 4 (2019), arXiv:1904.05363 [gr-qc].

[8] H. A. Buchdahl, Phys. Rev. 116, 1027 (1959).

[9] I. Goldman and S. Nussinov, Phys. Rev. D 40, 3221 (1989).

[10] A. Gould, B. T. Draine, R. W. Romani, and S. Nussinov, 
Phys. Lett. B 238, 337 (1990).

[11] A. Henriques, A. R. Liddle, and R. Moorhouse, Nucl. Phys. B 337, 737 (1990).

[12] A. Henriques, A. R. Liddle, and R. Moorhouse, Phys. Lett. B 233, 99 (1989).

[13] S. Leung, M. Chu, and L. Lin, Phys. Rev. D 84, 107301 (2011), arXiv:1111.1787 [astro-ph.CO].

[14] F. Di Giovanni, S. Fakhry, N. Sanchis-Gual, J. C. Degollado, and J. A. Font, (2020), arXiv:2006.08583 [gr-qc].

[15] R. L. Bowers and E. Liang, Astrophys. J. 188, 657 (1974).

[16] P. S. Letelier, Phys. Rev. D 22, 807 (1980).

[17] L. Herrera, A. Di Prisco, J. Martin, J. Ospino, N. Santos, and O. Troconis, Phys. Rev. D 69, 084026 (2004), arXiv:gr-qc/0403006.

[18] J. R. David, G. Mandal, and S. R. Wadia, Phys. Rept. 369, 549 (2002), arXiv:hep-th/0203048.

[19] I. Bena and N. P. Warner, Lect. Notes Phys. 755, 1 (2008), arXiv:hep-th/0701216.

[20] R. C. Myers, Gen. Rel. Grav. 29, 1217 (1997), arXiv:grqc/9705065.

[21] V. Balasubramanian, J. de Boer, S. El-Showk, and I. Messamah, Class. Quant. Grav. 25, 214004 (2008), arXiv:0811.0263 [hep-th].

[22] I. Bena and N. P. Warner, (2013), arXiv:1311.4538 [hepth].

[23] M. Visser, C. Barcelo, S. Liberati, and S. Sonego, PoS BHGRS, 010 (2008), arXiv:0902.0346 [gr-qc].

[24] P. Chen, W. G. Unruh, C.-H. Wu, and D.-H. Yeom, Phys. Rev. D 97, 064045 (2018), arXiv:1710.01533 [grqc].

[25] C. Berthiere, D. Sarkar, and S. N. Solodukhin, Phys. Lett. B 786, 21 (2018), arXiv:1712.09914 [hep-th].

[26] V. Baccetti, R. B. Mann, and D. R. Terno, Class. Quant. Grav. 35, 185005 (2018), arXiv:1610.07839 [gr-qc].

[27] D. Guerra, C. F. Macedo, and P. Pani, JCAP 09, 061 (2019), arXiv:1909.05515 [gr-qc].

[28] G. Grilli di Cortona, E. Hardy, J. Pardo Vega, and G. Villadoro, JHEP 01, 034 (2016), arXiv:1511.02867 [hep-ph].

[29] W. Hu, R. Barkana, and A. Gruzinov, Phys. Rev. Lett. 85, 1158 (2000), arXiv:astro-ph/0003365.

[30] L. Hui, J. P. Ostriker, S. Tremaine, and E. Witten, Phys. Rev. D 95, 043541 (2017), arXiv:1610.08297 [astroph.CO].

[31] B. Li, T. Rindler-Daller, and P. R. Shapiro, Phys. Rev. D 89, 083536 (2014), arXiv:1310.6061 [astro-ph.CO].

[32] V. H. Robles and T. Matos, Mon. Not. Roy. Astron. Soc. 422, 282 (2012), arXiv:1201.3032 [astro-ph.CO].

[33] N. Bar, D. Blas, K. Blum, and S. Sibiryakov, Phys. Rev. D 98, 083027 (2018), arXiv:1805.00122 [astro-ph.CO].

[34] L. Annulli, V. Cardoso, and R. Vicente, Phys. Rev. D 102, 063022 (2020), arXiv:2009.00012 [gr-qc].

[35] D. J. Kaup, Phys. Rev. 172, 1331 (1968).

[36] R. Ruffini and S. Bonazzola, Phys. Rev. 187, 1767 (1969).

[37] E. Seidel and W.-M. Suen, Phys. Rev. Lett. 72, 2516 (1994), arXiv:gr-qc/9309015.

[38] G. Narain, J. Schaffner-Bielich, and I. N. Mishustin, Phys. Rev. D 74, 063003 (2006), arXiv:astro-ph/0605724.

[39] M. Raidal, S. Solodukhin, V. Vaskonen, and H. Veermäe, Phys. Rev. D 97, 123520 (2018), arXiv:1802.07728 [astroph.CO].

[40] M. Deliyergiyev, A. Del Popolo, L. Tolos, M. Le Delliou, X. Lee, and F. Burgio, Phys. Rev. D 99, 063015 (2019),
arXiv:1903.01183 [gr-qc].

[41] S. L. Liebling and C. Palenzuela, Living Rev. Rel. 20, 5 (2017), arXiv:1202.5809 [gr-qc].

[42] G. F. Giudice, M. McCullough, and A. Urbano, JCAP 10, 001 (2016), arXiv:1605.01209 [hep-ph].

[43] N. Sanchis-Gual, F. Di Giovanni, M. Zilhão, C. Herdeiro, P. Cerdá-Durán, J. Font, and E. Radu, Phys. Rev. Lett. 123, 221101 (2019), arXiv:1907.12565 [gr-qc].

[44] M. Kesden, J. Gair, and M. Kamionkowski, Phys. Rev. D 71, 044015 (2005), arXiv:astro-ph/0411478.

[45] E. Berti et al., Class. Quant. Grav. 32, 243001 (2015), arXiv:1501.07274 [gr-qc].

[46] C. Bambi, Rev. Mod. Phys. 89, 025001 (2017), arXiv:1509.03884 [gr-qc].

[47] N. Sennett, T. Hinderer, J. Steinhoff, A. Buonanno, and S. Ossokine, Phys. Rev. D 96, 024002 (2017), arXiv:1704.08651 [gr-qc].

[48] C. F. Macedo, P. Pani, V. Cardoso, and L. C. B. Crispino, Phys. Rev. D 88, 064046 (2013), arXiv:1307.4812 [gr-qc].

[49] S. Yoshida, Y. Eriguchi, and T. Futamase, Phys. Rev. D 50, 6235 (1994).

[50] F. Guzman and J. Rueda-Becerril, Phys. Rev. D 80, 084023 (2009), arXiv:1009.1250 [astro-ph.HE].

[51] C. Palenzuela, L. Lehner, and S. L. Liebling, Phys. Rev. D 77, 044036 (2008), arXiv:0706.2435 [gr-qc].

[52] C. Palenzuela, I. Olabarrieta, L. Lehner, and S. L. Liebling, Phys. Rev. D 75, 064005 (2007), arXiv:grqc/0612067.

[53] M. W. Choptuik and F. Pretorius, Phys. Rev. Lett. 104, 111101 (2010), arXiv:0908.1780 [gr-qc].

[54] C. Palenzuela, P. Pani, M. Bezares, V. Cardoso, L. Lehner, and S. Liebling, Phys. Rev. D 96, 104058 (2017), arXiv:1710.09432 [gr-qc].

[55] T. Helfer, D. J. E. Marsh, K. Clough, M. Fairbairn, E. A. Lim, and R. Becerril, JCAP 03, 055 (2017), arXiv:1609.04724 [astro-ph.CO].

[56] T. Dietrich, S. Ossokine, and K. Clough, Class. Quant. Grav. 36, 025002 (2019), arXiv:1807.06959 [gr-qc].

[57] H. Olivares, Z. Younsi, C. M. Fromm, M. De Laurentis, O. Porth, Y. Mizuno, H. Falcke, M. Kramer, and L. Rezzolla, Mon. Not. Roy. Astron. Soc. 497, 521 (2020), arXiv:1809.08682 [gr-qc].

[58] N. Sanchis-Gual, M. Zilhão, C. Herdeiro, F. Di Giovanni, J. A. Font, and E. Radu, (2020), arXiv:2007.11584 [gr$\mathrm{qc}]$.

[59] M. Bezares, C. Palenzuela, and C. Bona, Phys. Rev. D 95, 124005 (2017), arXiv:1705.01071 [gr-qc].

[60] F. Di Giovanni, N. Sanchis-Gual, P. Cerdá-Durán, M. Zilhão, C. Herdeiro, J. Font, and E. Radu, (2020), arXiv:2010.05845 [gr-qc].

[61] R. Friedberg, T. Lee, and Y. Pang, Phys. Rev. D 35, 3658 (1987).

[62] B. Kleihaus, J. Kunz, and M. List, Phys. Rev. D 72, 064002 (2005), arXiv:gr-qc/0505143.

[63] B. Kleihaus, J. Kunz, M. List, and I. Schaffer, Phys. Rev. D 77, 064025 (2008), arXiv:0712.3742 [gr-qc].

[64] B. Kleihaus, J. Kunz, and S. Schneider, Phys. Rev. D 85, 024045 (2012), arXiv:1109.5858 [gr-qc].

[65] S. R. Coleman, Nucl. Phys. B 262, 263 (1985), [Erratum: Nucl.Phys.B 269, 744 (1986)].

[66] F. E. Schunck and D. F. Torres, Int. J. Mod. Phys. D 9, 601 (2000), arXiv:gr-qc/9911038.

[67] G. Choi, H.-J. He, and E. D. Schiappacasse, JCAP 10, 
043 (2019), arXiv:1906.02094 [astro-ph.CO].

[68] M. P. Hertzberg and E. D. Schiappacasse, JCAP 08, 028 (2018), arXiv:1804.07255 [hep-ph].

[69] S. Davidson and T. Schwetz, Phys. Rev. D 93, 123509 (2016), arXiv:1603.04249 [astro-ph.CO].

[70] T. Lee and Y. Pang, Nucl. Phys. B 315, 477 (1989).

[71] M. Gleiser, Phys. Rev. D 38, 2376 (1988), [Erratum: Phys.Rev.D 39, 1257 (1989)].

[72] M. Gleiser and R. Watkins, Nucl. Phys. B 319, 733 (1989).

[73] F. V. Kusmartsev, E. W. Mielke, and F. E. Schunck, Phys. Rev. D 43, 3895 (1991), arXiv:0810.0696 [astroph].

[74] T. Tamaki and N. Sakai, Phys. Rev. D 83, 044027 (2011), arXiv:1105.2932 [gr-qc].

[75] R. Sorkin, Astrophys. J. 249, 254 (1981).

[76] J. S. Schiffrin and R. M. Wald, Class. Quant. Grav. 31, 035024 (2014), arXiv:1310.5117 [gr-qc].

[77] E. Seidel and W.-M. Suen, Phys. Rev. D 42, 384 (1990).

[78] J. Balakrishna, E. Seidel, and W.-M. Suen, Phys. Rev.
D 58, 104004 (1998), arXiv:gr-qc/9712064.

[79] F. Guzman, Phys. Rev. D 70, 044033 (2004), arXiv:grqc/0407054.

[80] S. Valdez-Alvarado, C. Palenzuela, D. Alic, and L. Ureña-López, Phys. Rev. D 87, 084040 (2013), arXiv:1210.2299 [gr-qc].

[81] J. L. Friedman, Comm. Math. Phys. 63, 243 (1978).

[82] L. G. Collodel, B. Kleihaus, and J. Kunz, Phys. Rev. D 96, 084066 (2017), arXiv:1708.02057 [gr-qc].

[83] J. Keir, Class. Quant. Grav. 33, 135009 (2016), arXiv:1404.7036 [gr-qc].

[84] C. Reisswig, C. Ott, E. Abdikamalov, R. Haas, P. Moesta, and E. Schnetter, Phys. Rev. Lett. 111, 151101 (2013), arXiv:1304.7787 [astro-ph.CO].

[85] F. Pretorius, Class. Quant. Grav. 22, 425 (2005), arXiv:gr-qc/0407110 [gr-qc].

[86] W. E. East, F. Pretorius, and B. C. Stephens, Phys. Rev. D 85, 124010 (2012), arXiv:1112.3094 [gr-qc].

[87] L. Lindblom and B. Szilagyi, Phys. Rev. D80, 084019 (2009), arXiv:0904.4873 [gr-qc]. 